\title{
Multi-objective environmentally sustainable road network design using Pareto optimization
}

\author{
Yi Wang \\ Department of Civil Engineering, The University of Hong Kong, China \\ $\&$ \\ W. Y. Szeto* \\ Department of Civil Engineering, The University of Hong Kong, China \\ The University of Hong Kong Shenzhen Institute of Research and Innovation, Shenzhen, China
}

\begin{abstract}
In this paper, a bi-level transportation network design problem with environmental considerations is investigated. To explicitly reflect various requirements of environmental sustainability from planners, total emissions costs and total excessive noise cost are minimized along with total system travel time while performing optimal capacity expansion. To leave space for additional information on decision-making for planners and provide a more generalized description of solution optimality, the Pareto optimization approach is adopted. The study proposes a multi-objective variant of a new meta-heuristic named Chemical Reaction Optimization as the optimization tool to solve the formulated network design problem. Pareto front approximations have been successfully acquired for each scenario tested using the Sioux Falls network. The results show that the relations between pairwise objectives can change significantly (e.g., from conflicting to aligned) with regard to different demand situations. The newly proposed multi-objective metaheuristic succeeds to produce better approximations of the Pareto front than NSGA-II with comparable runtime for the considered road network.
\end{abstract}

\section{INTRODUCTION}

As a dramatic rise is observed in the amount of road traffic during the past decades, together with the mega expansion and construction of road transportation infrastructures being carried out to accommodate the rise, more and more outputs are emitted to the environment from road transportation, such as vehicular emissions and traffic noise. Scientific evidence of a potential causality between transportation and public health has been emerging as it relates to the effects of air pollutants on respiratory health and the community costs of noise (Banister, 2007). In recent years, the fact that road transportation negatively affects the quality of the environment and deteriorates its bearing capacity has drawn a wide range of concerns among researchers. Although the technologies for more environmentally friendly vehicles (e.g., fuel-efficient vehicles, electric automobiles, and cleaner fuels) and noise mitigation measures (e.g., design and installation of noise barriers and low-noise materials for road pavement) are advancing, tackling urban traffic-related environmental problems should be viewed within a broader perspective. Therefore, it is important for planners to be able to quantify the environmental impacts of road traffic during the planning of transportation system (López and Monzón, 2010; Ma et al., 2014). This brings about a variant of the classical road network design problem (NDP), namely the bi-level transportation network design problem with environmental considerations (BTPE), or equivalently the environmentally sustainable NDP, as defined and reviewed by Szeto et al. (2012). This new research area focuses on the optimal decision on road expansion or addition not only to minimize total system travel time (and its cost) but also to quantify and account for the effects of various dimensions of environmental sustainability (ES).

In many cities, noise and air quality levels are higher than their local standards, mainly due to vehicular emissions, energy use in commercial and residential buildings (see e.g., Wang and Adeli, 2014; Rafiei and Adeli, 2016), and traffic noise (see e.g., Akinyemi and Zuidgeest, 2002). Vehicular emissions are a commonly considered ES measure in BTPE studies, whose effects have been accounted for in either constraints or objective function. Mathew and Sharma (2006) considered vehicular emissions together with congestion in the user equilibrium constraint 
into the modeling of a continuous NDP. Zhao and Gao (2006) carried out a design for discrete BTPE by deploying an emission capacity constraint in the problem formulation. Szeto et al. (2015) formulated a sustainable road network design problem by restricting the overall vehicular emissions within the maximum acceptable target. While the efforts have been made on the identification of a conflicting relationship between reducing travel time and traffic emissions (e.g., Rilett and Benedek, 1994; Nagurney, 2000a, b; Yin and Lawphongpanich, 2006; Szeto et al., 2008; Ferguson et al., 2012), the BTPEs incorporating vehicular emission minimization spring up. Kim and Kim (2006) formulated an NDP model for multimodal networks, in which the objective is to minimize total social cost including an environmental cost. Qiu and Chen (2007) took the cost of environmental pollution into account by combining with other objectives such as travel time cost, investment cost, land use cost, and energy consumption cost in the design for a continuous NDP. Jia et al. (2009) proposed a model for a discrete NDP to minimize the yearly cumulative cost and the objective function consists of vehicular emission cost, total travel time cost, construction cost, maintenance cost, and energy consumption cost. Chen and $\mathrm{Xu}$ (2012) incorporated total travel time, carbon monoxide emissions, and spatial travel time equity under demand uncertainty into the upper level objective function of a bi-level model. Szeto et al. (2014) presented a discrete NDP model to deal with the minimization of total travel time cost, total vehicular emission cost, and total noise cost simultaneously. Jiang and Szeto (2015) proposed a timedependent discrete NDP that considers health impacts from road traffic emissions, noise, and accidents due to network expansion. Huang et al. (2015) proposed a method to maximize the traffic flow of road network with the noise capacity constraints in a road network. In the field of BTPE with dynamic traffic, Wismans et al. (2011a, b) contributed significantly to the investigation of the impacts from traffic externalities, including climate and noise, while seeking for optimal dynamic traffic measures. They further incorporated air quality and safety into the framework (Wismans et al., 2012), and provided ranking and pruning methods to better assist the decision-making process (Wismans et al., 2014b).

A number of observations can be drawn from the existing BTPE literature, wherein lies the motivation of this study. First, among above studies of BTPE, it is noticed that vehicular emissions are a widely considered dimension of ES but traffic noise is not. Sufficient scientific evidence has shown that exposure to noise constitutes human health risks, including performance reduction, hearing impairment, hypertension and ischemic heart disease (Linster, 1990; Passchier-Vermeer and Passchier, 2000). Motor vehicles usually are the primary source of noise disturbances at home (Delucchi and Hsu, 1996). Both Levinson et al. (1996) and Delucchi and Hsu (1996) pointed out that motor vehicle noise prominently affects the value of residential properties based on hedonic pricing, which could lead to a depreciation as high as $\$ 40$ billion per year in the USA (1990 data, 1991 dollars). These damages of housing value cast a profound influence on the land use pattern in a long run. Hence, traffic noise is worth emphasizing and investigating in traffic-related studies. It has been sketchily verified that there exist trade-offs among minimizing total travel time cost, total emission cost, and total noise cost (Szeto et al., 2014), which further endows a BTPE with a multi-objective nature. Indeed, the relationship between traffic noise and vehicular emissions has not been investigated in a multi-criteria BTPE, and only in monoobjective BTPE modeling as a constraint (e.g., Huang et al., 2009) or as a weighted attribute in the transformed single objective function (e.g., Szeto et al., 2014).

Second, the resolution of a multi-objective problem yields a set of compromise solutions, i.e., Pareto-optimal solutions, exhibiting the optimal trade-offs between objectives. In most BTPE studies, the weighted sum approach (or weighting method) has been adopted to transfer the multi-objective optimization problem into a mono-objective optimization problem by combining all objectives in a single objective function with a selected set of weights for objectives (e.g., Kim and Kim, 2006; Qiu and Chen, 2007; Jia et al., 2009). It is appreciated that this method contributes greatly to seeking to a single compromised solution for practical implementation and providing multiple solution points by varying the weights consistently. However, a fundamental deficiency in the weighted sum method is not able to depict the whole Pareto optimal set if the Pareto optimal hypersurface is not convex (Marler and Arora, 2010). Moreover, the selection of scalar weights for different objective attributes reflects presumably incorporated preferences and thus implants the decision-making process into the algorithm. These predefined weights result in equipping final solutions with the subjective perceptions from certain designers and the customizability for specific problems. To provide one generalized description of solution optimality and more detailed trade-offs between objectives, as well as leave space for additional information on decision-making for planners, solving a multi-objective model for BTPE by Pareto optimization is desiderated.

Concerning the solution method, meta-heuristics are earning popularity in handling bi-level NDPs or BTPEs, owing to their insensitivity to the mathematical property of the problem, such as non-convexity, nonlinearity, or discontinuity of the search space (Farahani et al., 2013). A number of metaheuristics or their hybrids have been extensively applied for tackling mono-objective NDPs and BTPEs, including genetic algorithm (GA) (e.g., Ukkusuri et al., 2007; $\mathrm{Ng}$ et al., 2009; Unnikrishnan and Lin, 2012), simulated annealing (SA) (e.g., Meng and Yang, 2002; Qin et al., 2013), particle swarm optimization (e.g., Wang et al., 
2015), ant colony optimization (e.g., Vitins and Axhausen, 2009), and artificial bee colony (e.g., Szeto et al., 2011, 2015). Inheriting the merits of meta-heuristics mentioned above, as well as being able to provide a set of solutions within a single run, multi-objective metaheuristics are proved suitable for finding multiple Pareto-optimal solutions, especially of sophisticated bi-level problems. In the literature, a limited number of studies have proposed or applied multi-objective metaheuristics to obtain satisfactory Pareto-optimal solutions to multi-objective NDPs or BTPEs. The fast elitist non-dominated sorting genetic algorithm (NSGA-II) is most widely adopted (e.g., Sharma and Mathew, 2011; Wismans et al., 2011a, b, 2012, 2014b; Brands and Berkum, 2014a). Research efforts have also been contributed to its variant or hybrid, such as the epsilon non-dominated sorting genetic algorithm ( $\varepsilon$-NSGAII) (e.g., Brands et al., 2014b) and multi-objective hybrid genetic algorithm (e.g., Miandoabchi et al., 2013). Besides NSGAII, the strength Pareto evolutionary algorithm 2 and 2+ (SPEA2 and SPEA2+) (e.g., Wismans et al., 2011a; 2011b; 2014a), the multi-objective evolutionary simulated annealing (e.g., Possel et al., 2010), and the multi-objective hybrid artificial bee colony algorithm (e.g., Miandoabchi et al., 2013) have also been successfully applied for tackling multi-objective NDPs or BTPEs with promising performance.

Recently, a new meta-heuristic named chemical reaction optimization (CRO) was proposed by Lam and $\mathrm{Li}$ (2010). Various mono-objective benchmark and real-world problems have been successfully solved by CRO in the literature (e.g., Xu et al., 2011; Lam et al., 2012a, b; Sun et al., 2012; Yu et al., 2012). A number of its hybrid variants have been reviewed by Siddique and Adeli (2017). Szeto et al. (2014) have further demonstrated its effectiveness and efficiency in solving BTPE. Its potential applications for large transportation networks are verified to be promising. Recently, Chaabani et al. (2014) firstly developed an indicator-based chemical reaction algorithm. Their experimental results have shown its superior performance on tackling a series of multi-objective benchmark problems compared with a number of multi-objective evolutionary algorithms. Their team further developed an efficient multiobjective chemical reaction optimization algorithm by proposing a new quasi-linear average time complexity quick non-dominated sorting algorithm (Bechikh et al., 2015). Meanwhile, Bouzoubia (2014) also proposed a multi-objective chemical reaction optimization algorithm for solving the well-known salesman problem with multiple objectives. In the field of magnetics, Duan and Hai (2014) proposed an orthogonal multi-objective chemical reaction optimization approach based on Pareto ranking scheme to handle the multi-objective optimization in the brushless DC motor design. More recently, $\mathrm{Li}$ et al. (2016) further developed a decomposition-based chemical reaction optimization algorithm and demonstrated its efficiency on solving 20 benchmark functions compared with existing techniques. However, there is no sign of applying this novel meta-heuristic in solving multi-objective BTPEs or NDPs in the literature reviewed. Therefore, the assessment of the performance of such CRO for this case is valuable. We are interested in developing a multi-objective version of CRO, named non-dominated sorting chemical reaction optimization (NSCRO), and exploiting its remarkable characteristics of chemical reactions for approximating the Pareto front of the proposed multi-objective BTPE.

The main contributions of this study lie in the following aspects: firstly, it proposes a new multi-objective model for BTPE which is able to provide environmentally conscious planners with a comprehensive set of Pareto solutions. The trade-offs between minimizing congestion, vehicular emissions, and noise are explicitly examined to account for the planners' desired objectives for different requirements. Secondly, a new multi-objective meta-heuristic NSCRO is developed and implemented for solving the proposed problem.

The remainder of this study is organized as follows. In the next section, the proposed bi-level tri-objective BTPE is mathematically formulated. Section 3 presents the solution method, i.e., non-dominated sorting chemical reaction optimization (NSCRO) in details. Section 4 is dedicated to the numerical studies on a benchmark transportation network, as well as the comparison of computational performance between NSCRO, NSGA-II, and the brute force method. Finally, Section 5 offers the conclusions and suggestions for future research.

\section{PROBLEM FORMULATION}

The proposed tri-objective BTPE is formulated as a bilevel problem within budget constraints. The lower level problem models travelers' route choice over an improved road network using the classic deterministic user equilibrium (DUE) concept. The upper level problem depicts the decision making on road expansions of environmentally conscious planners to minimize the total system travel time cost (TSTC), total vehicular emission cost (TEC) and total excessive noise cost $(T N C)$ at the same time. The mathematical details of these two subproblems will be given in Sections 2.1 and 2.2, respectively.

\subsection{Lower level problem}

The DUE concept is commonly used to model users' behavior in transportation planning (Sharma and Mathew, 2011). Though dynamic traffic assignment is capable of providing more detailed traffic patterns for estimating environmental impacts, the accuracy of the estimated traffic pattern highly depends on the accuracy of input data for model calibration and validation. For long term planning, very accurate input data is often unavailable. 
Hence, the DUE concept is deemed sufficient and appropriate for this study. The DUE concept is based on Wardrop's first principle, which states that no driver can unilaterally reduce his/her travel cost by shifting to another route. A DUE solution can be obtained by solving the following this equivalent nonlinear mathematical optimization program:

$$
\begin{array}{ll} 
& \min _{\mathbf{v}} \sum_{a \in A} \int_{0}^{v_{a}} t_{a}\left(x, u_{a}\right) d x \\
\text { subject to } & \sum_{r \in R_{w}} f_{r}=d_{w}, \forall w \in W, \\
& f_{r} \geq 0, \forall r \in R_{w}, w \in W, \\
& v_{a}=\sum_{r \in R} f_{r} \cdot \delta_{a r}, \forall a \in A,
\end{array}
$$

and $t_{a}$ is estimated by a widely used link performance function developed by U.S. Bureau of Public Roads (1964):

$$
t_{a}=t_{a}^{0} \cdot\left[1+0.15 \cdot\left(\frac{v_{a}}{c_{a}+u_{a}}\right)^{4}\right],
$$

where

$A$ : set of network links

$W$ : set of all origin-destination (O-D) pair

$R_{w}$ : set of routes $r$ between O-D pair $w$

$R$ : set of routes in the network, $R=\bigcup_{w \in W} R_{w}$

$v_{a}$ : flow on link $a$

$f_{r}$ : flow on route $r$

$d_{w}$ : hourly demand between O-D pair $w$ (fixed)

$\delta_{a r}$ : binary variable, which equals 1 if route $r$ uses link $a$, and 0 otherwise

$u_{a}$ : link capacity increment (fixed in the lower level problem)

$c_{a}$ : original link capacity of link $a$

$t_{a}$ : travel time on link $a$

$t_{0}$ : free flow travel time on link $a$

$\mathbf{v}$ : vector of link flows, $\mathbf{v}=\left(v_{a}\right)$

\subsection{Upper level problem}

From the perspective of planners, the upper level problem decides infrastructure measures to be implemented to optimize the objectives. Classically, the network design problem is concerned with building new streets or expanding the capacity of the existing streets (Dantzig et al., 1978). In this study, constant capacity expansion (e.g., lane addition) is considered to be the design measure. Hence, the decision variable in the upper level problem is the optimal road capacity expansion vector $\mathbf{u}$, consisting of the discrete capacity enhancement $u_{a}$ of each link. This decision variable the route choice of travelers and hence link flows $\mathbf{v}$ and therefore $\mathbf{v}$ can be expressed as $\mathbf{v}(\mathbf{u})$.

With the link flow vector $\mathbf{v}(\mathbf{u})$ from the lower level problem, this upper level problem aims to minimize TSTC, $T E C$, and TNC simultaneously, subject to a budget constraint. For practical reasons, the constraint implies that when the budget is limited, not all links can be expanded. Mathematically, the upper level is formulated as

$$
\begin{array}{ll}
\underset{\mathbf{u}}{\min }[\operatorname{TSTC}(\mathbf{u}, \mathbf{v}(\mathbf{u})), T E C(\mathbf{u}, \mathbf{v}(\mathbf{u})), T N C(\mathbf{u}, \mathbf{v}(\mathbf{u}))] & \\
\text { subject to } & \sum_{a \in A} g_{a}\left(u_{a}\right) \leq B,
\end{array}
$$

where

$u_{a}$ : decision variable, link capacity increment onto link $a$

$g_{a}$ : construction cost of link $a$ corresponding to the capacity-expansion scheme $u_{a}$, which is assumed to be proportional to the product of $u_{a}$ and the length of link $a$

$\mathbf{u}$ : vector of link capacity increments, $\mathbf{u}=\left(u_{a}\right)$

$B$ : total available budget for capacity enhancement

For each objective considered, the corresponding objective function is defined as follows:

Total system travel time cost (TSTC)

The total system travel time cost is defined by the product of total system travel time and the value of time (VOT):

$$
T S T C=V O T \cdot \sum_{a \in A} t_{a} \cdot v_{a}
$$

Total vehicular emission cost (TEC)

Three pollutants are considered in this study, due to their high shares of vehicular emissions and significant adverse effects on human health and ecological environment, including carbon monoxide (CO), violate organic compounds (VOC), and nitrogen oxides $\left(\mathrm{NO}_{\mathrm{x}}\right)$. A macroscopic TRANSYT-7F average-speed emission estimation model proposed by Penic and Upchurch (1992) is selected, which has been widely adopted in transportation studies (e.g., Tzeng and Chen, 1993; Rilett and Benedek, 1994; Benedek and Rilett, 1998; Wallace et al., 1998; Yin and Lawphongpanich, 2006; Nagurney et al., 2010; Li et al., 2012; Long et al., 2014; Szeto et al., 2014). The hourly total vehicular emission cost, denoted by $T E C$, is computed as the sum of the link-level emission cost of each pollutant $p$ along each link $a$ :

$$
T E C=\sum_{p} \sum_{a} \phi^{p} \cdot \frac{A^{p} \cdot e^{B^{p} \cdot \bar{s}_{a}}}{C^{p} \cdot \bar{S}_{a}} \cdot l_{a} \cdot v_{a}, \forall a, p,
$$

where

$p$ : pollutant, i.e., $\mathrm{CO}, \mathrm{NO}_{\mathrm{x}}$, and VOC

$l_{a}$ : link length of link $a(\mathrm{ft})$

$\bar{S}_{a}:$ average link travel speed (ft/s)

$v_{a}$ : flow on link $a(\mathrm{veh} / \mathrm{hr})$

$A^{p}, B^{p}$, and $C^{p}$ : constants

$\phi^{p}$ : monetary value $(\$ / \mathrm{g})$ for damage from pollutant $p$

The values for constants are obtained from Penic and Upchurch (1992) and Matthews (1999), listed in Table 1. In order to ensure formula consistency (Castillo et al., 2014a, b) and the results of the adopted estimation model interfacing the traffic model, the units of the coefficients are provided in Table 1 . The monetary value is converted to U.S. currency at its value in 1991 for consistency. Based on this estimation model, TEC is increasing with link traffic 
flow as long as the traveling speed is not too large (Yin and Lawphongpanich, 2006). With regard to non-zero average link speed, TEC decreases first and then increases (Wang and Szeto, 2017).

Table 1

Coefficients and monetary valuations of different pollutants

\begin{tabular}{llll}
\hline Pollutant & $\mathrm{CO}$ & $\mathrm{VOC}$ & $\mathrm{NO}_{\mathrm{x}}$ \\
\hline$A^{p}(\mathrm{~g} / \mathrm{ft}$ per veh $)$ & 3.3963 & 2.7843 & 1.5718 \\
$B^{p}(\mathrm{~s} / \mathrm{ft})$ & 0.014561 & 0.015062 & 0.040732 \\
$C^{p}(\mathrm{~s} / \mathrm{ft})$ & 1000 & 10000 & 10000 \\
$\phi^{p}(\$ /$ gram $)$ & 0.00051 & 0.00136 & 0.00103 \\
\hline
\end{tabular}

\section{Total noise cost (TNC)}

The estimations of traffic noise levels and the damage costs caused by traffic noise in terms of monetary valuation are both fundamental for modeling the economic damage of traffic noise pollution. It is widely agreed that in most situations, there is a nonzero threshold noise level below which little or no serious annoyance is caused, and hence noise has no cost (OECD, 1986). In this study, the impacts of excessive traffic noise above a threshold on residential property values are estimated by a general noise-cost model developed by Delucchi and Hsu (1996), which incorporates both a noise prediction model and a noise sensitivity depreciation index. This model is recognized as one of significant noise models reviewed by Litman (2003) and has been widely cited in reviews on the social costs of transportation in the U.S.

This model uses a simplified version of the well-known Federal Highway Administration's (FHWA's) Traffic Noise Model (TNM, formerly called "STAMINA"), which is recognized as one of the most commonly used noise prediction models in the review by Steele (2001) to calculate the equivalent hourly noise level from traffic. Then, it further monetizes the noise damages with an estimated housing value depreciation index from hedonic price analyses. The hourly total damage cost of excessive traffic noise $(T N C)$ directly from motor vehicles is estimated as follows:

$$
\begin{gathered}
T N C=\left(\sum_{a} A N_{a}\right) \cdot M \cdot P \cdot H V \cdot T, \\
A N_{a}=\frac{l_{a}}{5280} \cdot\left(\frac{\left(\int_{d_{e, a}}^{d_{t * a}, a} L_{e q}(w)_{a} d w\right)-A N B_{a}}{5280}\right), \forall a,
\end{gathered}
$$

where

$A N_{a}$ : area-noise level along link $a\left(\mathrm{dBA}-\mathrm{mi}^{2}\right)$

$M$ : density of housing units exposed to traffic noise above the threshold (housing units $/ \mathrm{mi}^{2}$ )

$P$ : median annualized value of housing units exposed to traffic noise above the threshold (\$/housing unit)

$H V$ : percentage of housing value lost for each decibel of noise above the threshold $\left(\mathrm{dBA}^{-1}\right)$

$T$ : dimensionless correction factor accounting for the cost of traffic noise away from one's home, based on time spent inside and outside one's home

$l_{a}$ : link length of link $a(\mathrm{ft})$

$d_{t^{*}, a}$ : equivalent distance from the center of the roadway of link $a$ to the point at which traffic noise drops to the threshold level (ft), calculated as the distance $d$ at $L_{e q}(d)_{a}=t^{*}$

$d_{e, a}$ : equivalent distance from the center of the roadway of link $a$ to the closest residence ( $\mathrm{ft}$ )

$t^{*}$ : threshold noise level below which the damage cost is presumed to be zero $(\mathrm{dBA})$

$A N B_{a}$ : area-noise level below the noise-damage threshold along link $a$ (dBA-ft), calculated as $t^{*}\left(d_{t^{*}, a}-d_{e, a}\right)$

$L_{e q}(d)_{a}$ : traffic noise $(\mathrm{dBA})$ as a function of distance $d$ from the road edge of link $a$. This function is integrated from the point $e$, at the closest residences, up to the point at which the noise level drops off to the threshold level $t^{*}$.

5280: $\mathrm{ft} / \mathrm{mile}$

The equivalent sound level function $L_{e q}$ of traffic noise along link $a$ with noise barriers is expressed as

$$
L_{e q}(d)_{a}=10 \cdot \log _{10}\left(0.0296 \cdot \frac{59}{180} \cdot v_{a} \cdot K_{a} \cdot\left(\frac{50}{d}\right)^{1.375}\right)-8.4, \forall a, h .
$$

where $K_{a}$ is the total noise-energy emissions. For illustration purposes, a simplified consideration for one vehicle type (i.e., light-duty automobile) on one road type (i.e., local roads) in urban areas is used. Based on Delucchi and Hsu (1996), the $K_{a}$ value in this study is calculated as

$$
K_{a}=\bar{S}_{a}^{3.174} \cdot 10^{0.115}+\frac{10^{5.6}}{\bar{S}_{a}} .
$$

It is known that accelerating vehicles are noisier than cruising vehicles due to higher engine rpm. The noiseenergy equation above includes an exponent (i.e., 5.6 in Equation (13)) that incorporates the consideration for traffic noise from both cruising and accelerating vehicles. On the roads where vehicles start and stop a lot with a low average speed (e.g., local roads), the cruising fraction is relatively low. According to Delucchi and Hsu (1996), assumptions are made that the cruising fraction is related to average speed in order to take the noise production during stop and go traffic into account aggregately. For more information of this exponent for different vehicles and road types, readers are welcome to consult Delucchi and Hsu (1996). According to Wang and Szeto (2017), the equivalent noise level increases first and then decreases with the average link speed.

The total noise cost is calculated as the product of the following components: 1) the excessive area-noise level $A N_{a}$ above a certain noise-damage threshold $t^{*}$, which equals the integral of $L_{e q}$ with respect to the width of area 
Table 2

Values of parameters for Sioux Falls (1990-1991 data) (Delucchi and Hsu, 1996)

\begin{tabular}{lllllll}
\hline Symbol & $d_{e}$ & $t^{*}$ & $H V$ & $T$ & $M$ & $P$ \\
Unit & $\mathrm{ft}$ & $\mathrm{dBA}$ & $\mathrm{dBA}^{-1}$ & Nil & housing units $/ \mathrm{mi}^{2}$ & \$/housing unit \\
\hline Value & 33.5 & 55 & 0.0085 & 1.272 & 1290.8 & 59100 \\
\hline
\end{tabular}

subject to traffic noise, minus the area-noise level below the noise-damage threshold $A N B_{a}$. When $d_{t^{*}}$ is less than $d_{e, a}$, it is assumed that there are no noise damages along that link, 2) the density of housing units $M$ subject to excessive noise and the median annualized value of housing units $P$. Due to the lack of data on housing density along specific types of roads, the smallest possible unit of analysis is the urbanized area and consequently Delucchi and Hsu (1996) estimated a single uniform density for each urbanized area, 3) the diminution in annualized housing value per excessive decibel $H V$, and 4) the parameter $T$ for scaling up noise cost caused at places other than residential property. For numerical results and analysis, a real network of Sioux Falls, South Dakota, USA is selected (see the Appendix). The parameter values are provided in Table 2 .

\section{SOLUTION METHOD}

The newly invented meta-heuristic chemical reaction optimization (CRO) ( $\mathrm{Lam}$ and $\mathrm{Li}, 2010$ ) exploits the conservation of energy and lower-energy-seeking principles of chemical reactions to solving optimization problems. In this study, a multi-objective variant of CRO named Non-dominated Sorting CRO (NSCRO) is proposed to tackle the formulated multi-objective BTPE, owing to its following characteristics: on one hand, CRO is a population-based metaheuristic that can manipulate a number of solutions within a single run, which provides high feasibility of employment of Pareto-ranking scheme. On the other hand, the relation between reactants and resultants in chemical reactions echoes that between parents and children in genetic algorithm (GA), which possesses successful multi-objective variants such as NSGA and NSGA-II. Besides previously listed BTPEs solved by GA, it also has a wide range of successful applications in various fields including structural engineering (e.g., Adeli and Cheng, 1993; Sarma and Adeli, 2001; Kim and Adeli, 2001; Sarma and Adeli, 2002, 2003; Kociecki and Adeli, 2013, 2014, 2015) and electrical and electronic engineering (e.g., Hung and Adeli, 1994). Owing to its friendly framework, GA allows other techniques to be easily incorporated to produce a hybrid that achieves the best performance on different problems (e.g., Adeli and Cheng, 1994a, b; Adeli and Kumar, 1995a, b; 1999; Sarma and Adeli, 2000a, b; Jiang and Adeli, 2008). These facts offer the inspiration and a basic framework for the development of NSCRO based on the multi-objective variant of GA.

In the following subsections, the detailed solution representation for the proposed BTPE is firstly provided, followed by a brief review of the basic features and algorithm design of original CRO. These related works provide fundamentals for the development of NSCRO in the last subsection.

\subsection{Solution representation}

For illustration purposes, constant capacity increment (lane addition) is considered to be the improvement strategy. Hence, expanding a link means that a constant of 1800 $\mathrm{veh} / \mathrm{hr}$ is added to the link $\left(u_{a}=1800 \mathrm{veh} / \mathrm{hr}\right)$; otherwise, no improvement on link capacity $\left(u_{a}=0 \mathrm{veh} / \mathrm{hr}\right)$. All the links in the network are allowed to be improved as long as the budget constraint is met. Hence, the solutions can be encoded as strings of binary variables, with the length equal to the total number of links in the network. Each bit corresponds to one network link, indicating whether the link should be expanded or not, i.e., 1 for $1800 \mathrm{veh} / \mathrm{hr}$ capacity enhancement and 0 for no capacity expansion. It is straightforward to consider a more generalized case by adopting a non-negative integer for each bit in the representation. However, this is not our main focus and hence we leave this for future implementation.

The initialization of population utilizes a random generator. For each bit of the string, a random number $k$ is generated from $[0,1]$. If $k$ is larger than 0.5 , the value of that bit is set to 1 . Otherwise, it is set to be equal to 0 . The same initial population generalization is used for both algorithms.

A repair process is specially designed for solving this BTPE. It ensures that the capacity improvement strategy represented by the solution is feasible, which satisfies the budget constraint (7). This method is implemented not only in the initialization stage but also to each new solution obtained by chemical reaction operators. The repair mechanism is to rescind the improvement of most costly links until it meets the budget constraint. Moreover, to ensure the effectiveness of solution operators, they are designed to guarantee that the changes made to solutions are not revoked by the repair process.

\subsection{Basic features of CRO}

This novel meta-heuristic mimics the process of a certain number of molecules in a closed container taking part in various chemical reactions to become the final resultants with the most stable states with the lowest free energy. The key concept of CRO is the conservation of energy in chemical reactions. Energy cannot be created or destroyed but is allowed to convert between different types or 
redistribute among different molecules through a series of elementary reactions, which inspires the CRO mechanism design.

The chemical process involves two major components to accomplish, namely, molecules and elementary reactions. Each molecule is endowed with several key chemical attributes, which are designed to represent essential operational features correspondingly, including a) molecular structure (denoted as $\omega$ ), representing a solution of the optimization problem; b) potential energy $(P E)$, corresponding to the objective value of the solution (to be minimized); and c) kinetic energy $(K E)$, indicating the tolerance for a new solution with worse fitness value than the existing one. Changes in $P E$ and $K E$ take place due to the changes of molecular structures through a series of chemical reactions. Four types of elementary reactions are simulated in the original CRO mechanism proposed by Lam and $\mathrm{Li}$ (2010), namely a) on-wall ineffective collision, a mild reaction where a molecule hits the wall of the container and then bounce away; b) decomposition, a vigorous reaction of one molecule decomposing into two after it hits the wall; c) intermolecular ineffective collision, a mild collision where two molecules collide with each other and then bounce away; and d) synthesis, the union of two molecules into one new resultant. As shown in Table 3 (second column), four chemical reaction search operators are designed in accordance with the intensity of reactions to seek for new solutions by altering molecular structures into different extent. The search operators in vigorous reactions (e.g., decomposition and synthesis) allow the algorithm to explore solutions in a region farther away from the existing ones in a solution space, while the search operators in mild collisions (e.g., on-wall ineffective collision and intermolecular collision) focus on neighborhood search in a solution space. Taking the solution encoded as strings of binary variables as an example, the mechanism of randomly flipping one bit on the string is used for the neighborhood operators in mild collisions; the mechanism of randomly splitting the string(s) into two segments with further regeneration or combination of segments is used for decomposition or synthesis operators.

After the variation of molecular structure(s), the following general rule is examined to determine if the elementary reaction can be realized or not:

$$
\underbrace{\sum_{\omega} P E_{\omega}+\sum_{\omega} K E_{\omega}}_{\text {reactants }}-\underbrace{\sum_{\omega^{\prime}} P E_{\omega^{\prime}}}_{\text {resulatnts }} \geq 0 .
$$

As provided in Table 3 (third column) in greater details, the respective criteria for each reaction to take place are set up using the $P E$ and $K E$ of reactant(s), the $P E$ of resultant(s), and sometimes buffer, which is the energy stored in the environment (i.e., container). Exemplified with the elementary reaction Decomposition, the criteria are illustrated as follows: firstly, the total energy of the reactant (sum of $P E_{\omega}$ and $K E_{\omega}$ ) is compared with the sum of the two resultants' potential energy: $P E_{\omega_{1}^{\prime}}$ and $P E_{\omega_{2}^{\prime}}$, calculated based on the varied molecular structures. If the former is larger, the reaction occurs. However, the reactant sometimes may not possess enough total energy to sustain such transformation. If so, a certain portion of energy in buffer accumulated from on-wall ineffective collisions can be utilized to support the change and the second condition is checked. If the second condition for occurrence is fulfilled, the elementary reaction is also allowed to take place, wherein the resultant(s) will be generated and replace the reactant(s). Otherwise, the resultant(s) cannot be generated and the reactant(s) remain unchanged. It is worth noting that the selection of new solutions is accomplished within the realization of the corresponding elementary reaction.

In the realization of reactions, the resultants' kinetic energy and the free energy buffer are updated via energy management rules (Table 3, last column). These rules guide the redistribution of energy among molecules as well as between molecules and the environment and guarantee the conservation of energy. Hence, the sum of energy before and after the reaction remains the same.

Similar to other meta-heuristics, CRO algorithm consists of three stages: initialization, iterations, and a final stage. The first stage is carried out by setting the population size of the parent population $N$, the initial $K E$ of molecules, and some system parameters including MoleColl, indicating the probability of the occurrence of an inter-molecular reaction, and KELossRate (defined below Table 3). Then, a sequence of iterations is performed until a stopping criterion is met. Within each iteration, a certain type of elementary reactions is selected to be undertaken through a series of predesigned criteria: firstly, an elementary collision type is selected, i.e., unimolecular collision (i.e., on-wall ineffective collision or decomposition) or inter-molecular collision (intermolecular ineffective collision or synthesis). This is carried out by generating a random number in the interval $[0,1]$. If the random number is larger than MoleColl, a unimolecular collision will occur. Otherwise, an intermolecular collision will take place. Next, pre-set criteria are used to proceed to decomposition and synthesis reactions. Lam and $\mathrm{Li}$ (2010) emphasized that these changeable criteria, as well as mechanisms for generating new solutions in each chemical reaction operators, empower CRO applicable for a wide range of optimization problems. We adopt the same decomposition and synthesis criteria initially proposed by Lam and Li (2010), wherein two pre-defined parameters $\alpha$ and $\beta$ are used. The decomposition criterion is that the selected molecule has not changed to a lower energy state for certain time, i.e., the number of collisions wherein the molecule did not change into a solution with lower $P E$ is greater than $\alpha$. The synthesis criterion is that both the selected molecules have insufficient $K E$, i.e. $K E_{\omega_{1}}<\beta$ and $K E_{\omega_{2}}<\beta$. 
Table 3

Elementary reactions in $\mathrm{CRO}$

\begin{tabular}{|c|c|c|c|}
\hline Reaction & Search operator & Condition(s) for occurrence & Energy management laws \\
\hline $\begin{array}{l}\text { On-wall } \\
\text { ineffective } \\
\text { collision }\end{array}$ & $\begin{array}{l}\text { Neighbourhood operator } \\
\text { e.g., one random flip in } \\
\text { the string } \\
{[1, \mathbf{0}, 0,1,] \rightarrow[1, \mathbf{1}, 0,1]}\end{array}$ & $P E_{\omega}+K E_{\omega} \geq P E_{\omega^{\prime}}$ & $\begin{array}{l}K E_{\omega^{\prime}}=\left(P E_{\omega}-P E_{\omega^{\prime}}+K E_{\omega}\right) \cdot q \\
\text { buffer }=\text { buffer }+\left(P E_{\omega}-P E_{\omega^{\prime}}+K E_{\omega}\right) \cdot(1-q)\end{array}$ \\
\hline Decomposition & $\begin{array}{l}\text { Decomposition operator } \\
\text { e.g., one random cut in } \\
\text { the string and split the } \\
\text { string into two with } \\
\text { random regeneration } \\
{[1,0,0,1,] \rightarrow[\mathbf{1}, \mathbf{0}, \mathbf{0}, \underline{1}]} \\
+[1,1,0, \mathbf{1}]\end{array}$ & $\begin{array}{l}P E_{\omega}+K E_{\omega} \geq P E_{\omega_{1}^{\prime}}+P E_{\omega_{2}^{\prime}} \\
\text { otherwise, } \\
P E_{\omega}+K E_{\omega}+\text { buffer } \geq P E_{\omega_{1}^{\prime}}+P E_{\omega_{2}^{\prime}}\end{array}$ & $\begin{array}{l}K E_{\omega_{1}^{\prime}}=\left(P E_{\omega}+K E_{\omega}-P E_{\omega_{1}^{\prime}}-P E_{\omega_{2}^{\prime}}\right) \cdot k, \\
K E_{\omega_{2}^{\prime}}=\left(P E_{\omega}+K E_{\omega}-P E_{\omega_{1}^{\prime}}-P E_{\omega_{2}^{\prime}}\right) \cdot(1-k) \text { or } \\
K E_{\omega_{1}^{\prime}}=\left(P E_{\omega}+K E_{\omega}-P E_{\omega_{1}^{\prime}}-P E_{\omega_{2}^{\prime}}+\text { buffer }\right) \cdot m_{1} \cdot m_{2}, \\
K E_{\omega_{2}^{\prime}}=\left(P E_{\omega}+K E_{\omega}-P E_{\omega_{1}^{\prime}}-P E_{\omega_{2}^{\prime}}+\text { buffer }-K E_{\omega_{1}^{\prime}}\right) \cdot m_{3} \cdot m_{4}\end{array}$ \\
\hline $\begin{array}{l}\text { Intermolecular } \\
\text { ineffective } \\
\text { collision }\end{array}$ & $\begin{array}{l}\text { Neighbourhood operator } \\
\text { e.g., apply the neighbor } \\
\text { operator adopted in on- } \\
\text { wall infective collision } \\
\text { twice simultaneously. }\end{array}$ & $P E_{\omega_{1}}+P E_{\omega_{2}}+K E_{\omega_{1}}+K E_{\omega_{2}} \geq P E_{\omega_{1}^{\prime}}+P E_{\omega_{2}^{\prime}}$ & $\begin{array}{l}K E_{\omega_{1}^{\prime}}=\left(P E_{\omega_{1}}+P E_{\omega_{2}}+K E_{\omega_{1}}+K E_{\omega_{2}}-P E_{\omega_{1}^{\prime}}-P E_{\omega_{2}^{\prime}}\right) \cdot k \\
K E_{\omega_{2}^{\prime}}=\left(P E_{\omega_{1}}+P E_{\omega_{2}}+K E_{\omega_{1}}+K E_{\omega_{2}}-P E_{\omega_{1}^{\prime}}-P E_{\omega_{2}^{\prime}}\right) \cdot(1-k)\end{array}$ \\
\hline Synthesis & 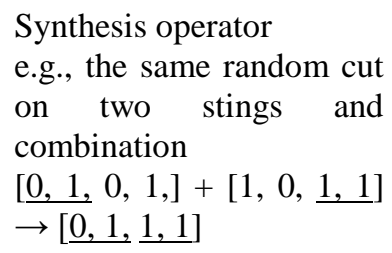 & $P E_{\omega_{1}}+P E_{\omega_{2}}+K E_{\omega_{1}}+K E_{\omega_{2}} \geq P E_{\omega^{\prime}}$ & $K E_{\omega^{\prime}}=P E_{\omega_{1}}+P E_{\omega_{2}}+K E_{\omega_{1}}+K E_{\omega_{2}}-P E_{\omega^{\prime}}$ \\
\hline
\end{tabular}

$\omega, \omega_{1}$, and $\omega_{2}$ are molecular structures and $\omega^{\prime}, \omega_{1}^{\prime}$, and $\omega_{2}^{\prime}$ are their variants after the reaction, respectively. $q$ is randomly generated from [KELossRate, 1], where KELossRate is a control parameter, i.e., the lower bound of the portion of KE not being lost to the environment.

$k, m_{1}, m_{2}, m_{3}$, and $m_{4}$ are independently and randomly generated from $[0,1]$. 
Whenever an elementary reaction is selected, if the conditions for occurrence based on energy (as shown in Table 3) are satisfied, the reaction will take place, wherein the molecules and their corresponding energies are updated based on energy management laws (as shown in Table 3). Otherwise, the corresponding reaction will not be realized and the molecules will remain exactly the same. The whole evolution process stops when the stopping criterion is met and the best solution is reported in the final stage. For more detailed algorithm design and parameter setting, readers are invited to consult the study by Lam and Li (2010).

\subsection{NSCRO}

In this section, the algorithm design of a nondominated sorting CRO (NSCRO) is presented. The main framework adopted from NSGA-II is introduced briefly, followed by some characteristic features designed in NSCRO, and the overall algorithm is presented in a flowchart in the end, with a comparison to NSGA-II.

\subsubsection{Main framework adopted from NSGA-II}

Besides tackling the multi-objective NDPs and BTPEs, NSGA-II has been successfully applied or hybridized to numerous optimization applications in civil engineering, including transportation engineering (e.g., Unnikrishnan et al., 2009; Bai et al., 2015) and structural engineering (e.g., Cha and Buyukozturk, 2015; Park et al., 2015; Oh et al., 2017). In virtue of the inherent similarity between the parent-children relationship in GA and reactant-resultant relationship in CRO, NSCRO draws the main framework from the Pareto-dominance based fast elitist non-dominated sorting genetic algorithm (NSGA-II) by Deb et al. (2002).

The main framework adopted from NSGA-II is illustrated in Figure 1. Initially, an offspring population (i.e., resultant population) $\mathbf{Q}_{\text {iter }}$ is obtained from a parent population (i.e., reactant population) $\mathbf{P}_{\text {iter }}($ size $=N)$ by operators, e.g., crossover and mutation operators in NSGA-
II. For more details on genetic operators, readers are welcome to consult Adeli and Hung (1995), Adeli and Sarma (2006), and Siddique and Adeli (2013). Once $\mathbf{Q}_{i t e r}$ is generated, a combined population $\mathbf{R}_{\text {iter }}$ is constructed by combining $\mathbf{P}_{\text {iter }}$ and $\mathbf{Q}_{\text {iter }}$. Then, the fast non-dominated sorting and crowding distance sorting proceed successively to rank all the solutions into each front $\left(F_{1}, F_{2}, F_{3}, \ldots, F_{n}\right.$, where $n$ is the total number of nonempty Pareto fronts classified). In order to avoid tautology, the procedure of the non-dominated sorting method can be referred to the studies by Goldberg (1989), Srinivas and Deb (1994), and Deb et al. (2002). In the end, the new parent population $\boldsymbol{P}_{\text {iter }+1}$ is constructed based on elitism by filling up with the top $N$ solutions.

NSCRO also adopts the framework in Figure 1. However, NSCRO differs from NSGA-II in several aspects. In the following section, three key components of NSCRO will be discussed to further distinguish NSCRO from NSGA-II in offspring population generation and solution fitness evaluation.

\subsubsection{Key component in NSCRO}

3.3.2.1 Probation system for reaction realization

Recall that in each chemical reaction in $\mathrm{CRO}$, the acquisition of new solutions is completed together with the replacement of old solutions within the realization of each reaction, rather than afterward. However, the construction of $\mathbf{R}_{\text {iter }}$ and non-dominated sorting requires the coexistence of both reactants (analogy to parents) and resultants (analogy to offspring). Hence, a probation system is proposed in order to adapt to the above framework.

This probation system is designed to postpone the realization of every chemical reaction in each iteration, by allowing temporary retention of all the molecules (solutions) including involved reactants in $\mathbf{P}_{\text {iter }}$ and generated resultants in $\mathbf{Q}_{\text {iter }}$ in each iteration. In NSCRO, an iteration completes until all the molecules in $\mathbf{P}_{\text {iter }}$ participate

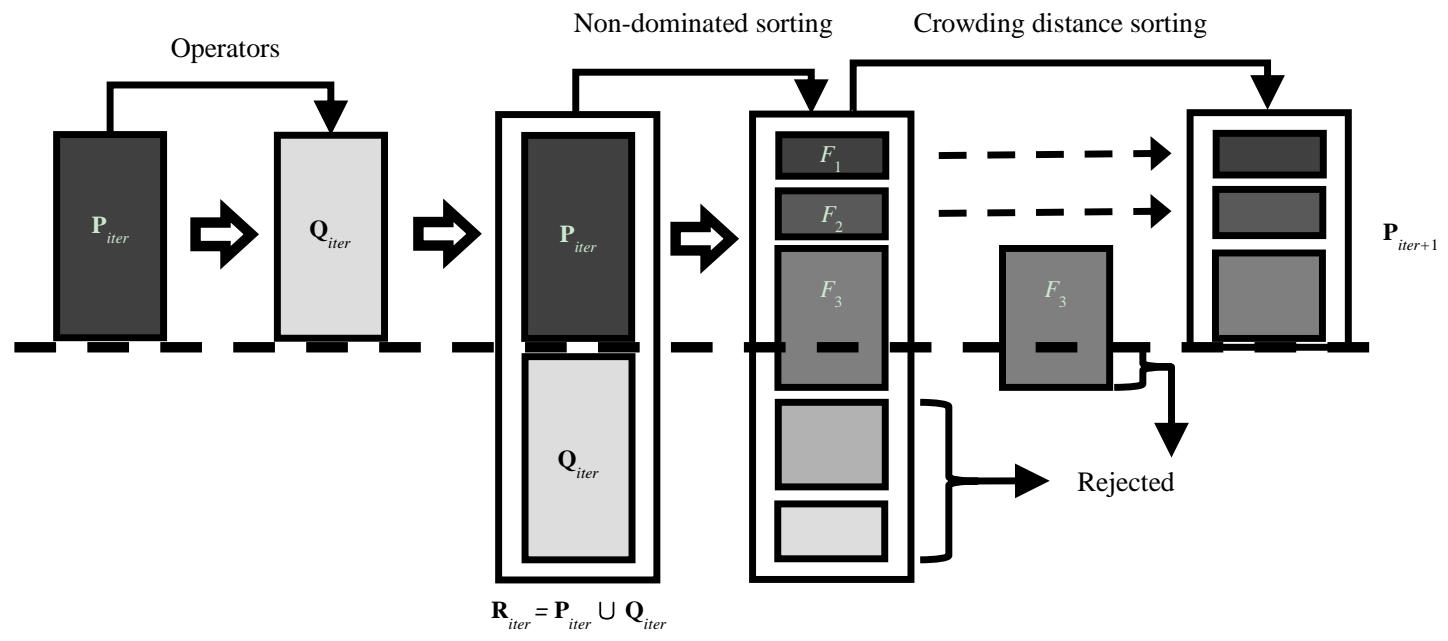

Fig. 1. Main framework of NSGA-II 
in one collision. In order to guarantee strict execution of energy conservation and management laws afterward, the details of the occurrence of each reaction are recorded in this system in order, including reaction type, reactants, and resultants. After the non-dominated sorting and crowding distance sorting, the $P E$ values of all the molecules can be computed accordingly, which will be detailed in the next subsection. With the $P E$ values of all the molecules, the realization of each recorded reaction can resume. Each reaction is checked by its corresponding condition(s) for the occurrence (Table 3). Once the conditions for occurrence are fulfilled, the temporarily reserved resultants will replace the reactants as finalized resultants, followed by energy redistribution based on corresponding energy management laws (Table 3). However, those reactions which cannot meet their corresponding condition(s) for occurrence are not allowed to occur, wherein the temporarily reserved resultants from this reaction will not be allowed to be produced. These temporarily reserved but not finalized resultants are defined as forbidden solutions and removed from the offspring population.

This probation system helps exploit the solution selection mechanism of CRO to multi-objective optimization and preserves the core concept of original CRO that energy conservation between reactants and finalized resultants is satisfied.

\subsubsection{2 $P E$ assignment based on Pareto dominance relation}

The $P E$ value of a molecule in NSCRO should be able to reflect the solution quality with a comprehensive consideration of multiple objectives simultaneously. The resolution of a multi-objective problem yields a set of compromise solutions, i.e., Pareto-optimal solutions, exhibiting the optimal trade-offs between objectives. To calculate the fitness value of each solution and distinguish between different solutions, Pareto dominance relation plays a prominent role. In NSGA-II, the quality of the solution is evaluated based on its non-dominance rank ( 1 is the best rank). All solutions in the same front have the same rank. Within each Pareto front, another measure named crowding distance is estimated to indicate the solution density surrounding a particular point (for the details of the calculation, please kindly refer to Deb et al., 2002). A larger crowding distance means a lower solution density, which will result in better diversity in a population (Deb et al., 2002). Therefore, between two solutions with different nondomination ranks, the solution with a lower non-dominance rank is preferred. If both solutions lie in the same front (i.e., they have the same rank), the solution with a larger crowding distance is preferred. These two measures jointly direct the search towards a uniformly spread out Paretooptimal front.

Based on these two comparison measures in NSGA-II, a formula for $P E$ assignment is proposed to preserve the Pareto dominance relations in the evaluation mechanism of
NSCRO. Algebraically, the $P E$ of each solution $\omega$ is calculated as follows:

$$
P E_{\omega}=\operatorname{rank}(\omega)+\frac{\delta}{1+\operatorname{crowd}(\omega)},
$$

where $\operatorname{rank}(\omega)$ and $\operatorname{crowd}(\omega)$ are the Pareto non-dominance rank and the crowding distance of solution $\omega$ defined in NSGA-II, respectively. The parameter $\delta$ here possesses a positive value from $(0,1)$. The value of 1 is excluded because it does not guarantee the dominance relationship when identical solutions are found (crowding distance may equal to 0 ). In this study, $\delta$ is set to 0.999 .

It is not difficult to prove that the function is monotone increasing with $\operatorname{rank}(\omega)$ and strictly decreasing with $\operatorname{crowd}(\omega)$. Moreover, the value of the second part can be proved to always fall in $(0,1)$, since the value of crowding distance is always non-negative. Hence, this formula assigns lower $P E$ to the solution with a smaller Pareto front rank and the solution with a larger value of crowding distance (i.e., located in a less crowded region) in a front. In summary, the Pareto dominance relations are fully embodied in the proposed formula for $P E$ assignment.

\subsubsection{Elitism in NSCRO}

In NSGA-II, solutions forming the new parent population $P_{\text {iter }+1}$ for the next iteration are chosen in a descending order of fitness based on the Pareto front rankings and crowded comparison operator. Since all parent and offspring populations $\mathbf{P}_{\text {iter }}$ and $\mathbf{Q}_{i t e r}$ are included in this set, elitism is ensured to prevent the loss of good solutions and achieve better convergence. Inspired by the elitist selection embedded in NSGA-II, as well as a prototype in chemical reactions, named catalyst, an elitist approach is proposed for this NSCRO

According to Section 3.3.2.1, any temporarily reserved solution in the probation system could bear the risk of being removed afterward due to a possible violation of reaction criteria. The algorithm's efficiency of approximating Pareto-optimal front would have been reduced, due to the possibility of each solution being currently or globally Pareto optimal but no longer acquired by the next parent population. Therefore, emphasis should be laid on the first non-dominated front after each non-dominated sorting, where the current Pareto optimal solutions reside. These solutions are very likely to continue being non-dominated solutions along with the approximation of Pareto front since they could be the Pareto optimal solutions forming the final Pareto front. These characteristics are analogous to the peculiarities possessed by a catalyst in a chemical reaction to some extent. The catalysts help increase the rate of a chemical reaction and are not consumed but recycled after the reaction. Therefore, the molecules representing nondominated solutions discovered in the current iteration are identified as catalysts and are designed to be remained to participate in the next round of chemical reactions. 
Therefore, elitism built in NSCRO aims to maintain the current non-dominated solutions in the parent population for further iterations, and it works as follows: to prepare the next parent population $\mathbf{P}_{\text {iter }+1}$ by the end of each iteration, two sources of solutions are selected successively: Firstly, duplicate all the solutions belonging to the first Pareto front (i.e., catalysts) in the current iteration from non-dominated sorting; followed by selecting all the finalized solutions (resultants) stored in the second front, the third front, and so on in the ascending order of $P E$ values until the number of solutions reaches the predefined population size $N$. Under particular circumstances, if the solution is identified as both a catalyst and a forbidden solution, it is duplicated into the next parent population and the original forbidden solution in the offspring population will still be removed when resuming the reactions. The duplication can be regarded as the regular addition of fresh catalysts to maintain constant activity in the chemical industry. Hence, this regular adjustment of the molecules in the container for chemical reactions does not have to break the laws of energy conservation. The laws of energy conservation are always witnessed from the beginning till the end of realization of all the reactions within each iteration.

\subsubsection{Overall procedure and comparison with NSGA-II}

The overall procedure of NSCRO is as follows. After the initialization of the parent population $\mathbf{P}_{\text {iter }}$, an offspring population $\mathbf{Q}_{\text {iter }}$ is obtained by applying the chemical reaction operators. With the presence of proposed probation system, the $\mathbf{P}_{\text {iter }}$ and $\mathbf{Q}_{\text {iter }}$ populations are not updated by energy management laws yet. Once all the solutions in $\mathbf{P}_{\text {iter }}$ have been processed by different operators, the offspring population $\mathbf{Q}_{\text {iter }}$ is generated. A combined population $\mathbf{R}_{\text {iter }}$ is constructed by combining $\mathbf{P}_{\text {iter }}$ and $\mathbf{Q}_{\text {iter }}$. The fast non-dominated sorting and crowding distance sorting are performed for this set, followed by $P E$ value assignment for each individual in $\mathbf{R}_{\text {iter }}$. The catalysts (the first non-dominated solutions) are easily identified after the sorting. Elitism is promised by performing step 11 . Then, the realizations of chemical reactions resume by examining the criteria and implementing energy management laws accordingly in step 12. Afterward, $\mathbf{R}_{\text {iter }}$ is updated by removing forbidden solutions sought from failed reactions. Finally, the construction of a parent population $\mathbf{P}_{\text {iter }+1}$ to be optimized in a new iteration is finished by step 13 .

\section{The NSCRO:}

Input: problem specific information (number of objectives, number of decision variables, objective functions)
1. Assign the parameter values

2. Set the iteration counter iter $=0$

3. Create a parent population $\mathbf{P}_{\text {iter }}$ and repair infeasible solutions

4. Do while (number of the lower level problems solved $<$ maximum number)

5. Do while (NOT all the solutions in $\mathbf{P}_{i t e r}$ are selected), apply chemical reaction operators to $\mathbf{P}_{i t e r}$ to form an offspring population $\mathbf{Q}_{\text {iter }}$
$5.1 \quad$ Generate $\lambda$ randomly from $[0,1]$
5.2 If $\lambda>$ MoleColl then
$5.3 \quad$ Select one molecule from $\mathbf{P}_{\text {iter }}$ randomly
5.4 If decomposition criterion is satisfied
then
5.5
5.6
5.7
5.8
5.9
5.10
5.11
5.12
5.13
5.14
Implement decomposition operator
Else
Implement neighborhood operator End if
Else
Select two molecules from $\mathbf{P}_{\text {iter }}$ randomly
If the synthesis criterion is satisfied then
Else Implement synthesis operator

twice simultaneously

5.15 End if

$5.16 \quad$ End if

5.17 Repair infeasible solution(s)

5.18 Update $\mathbf{Q}_{\text {iter }}$ by adding new solution(s)

sought from chemical reaction operators

5.19 Update chemical reaction record (reaction type, reactants, and resultants)

6. $\quad$ End do

7. $\quad \mathbf{R}_{\text {iter }}=\mathbf{P}_{\text {iter }} \cup \mathbf{Q}_{\text {iter }}$

8. Implement fast non-dominated sorting and crowding distance sorting to individuals in $\mathbf{R}_{\text {iter }}$

9. Compute the $P E$ value for each individual in

$\mathbf{R}_{\text {iter }}$

10. Catalysts (non-dominated solutions) identified

11. Fill $\mathbf{P}_{\text {iter }+1}$ with catalysts

12. Update $\mathbf{R}_{\text {iter }}$ by resuming the recorded

chemical reactions (examining the criteria and implementing energy management laws) in order.

13. Fill $\mathbf{P}_{i t e r+1}$ by adding a total of $N$ solutions stored in the second front, the third front and so on from $\mathbf{R}_{\text {iter }}$ in the ascending order of $P E$ values
14. iter $=$ iter +1
15. End do

Output: Pareto front approximation 


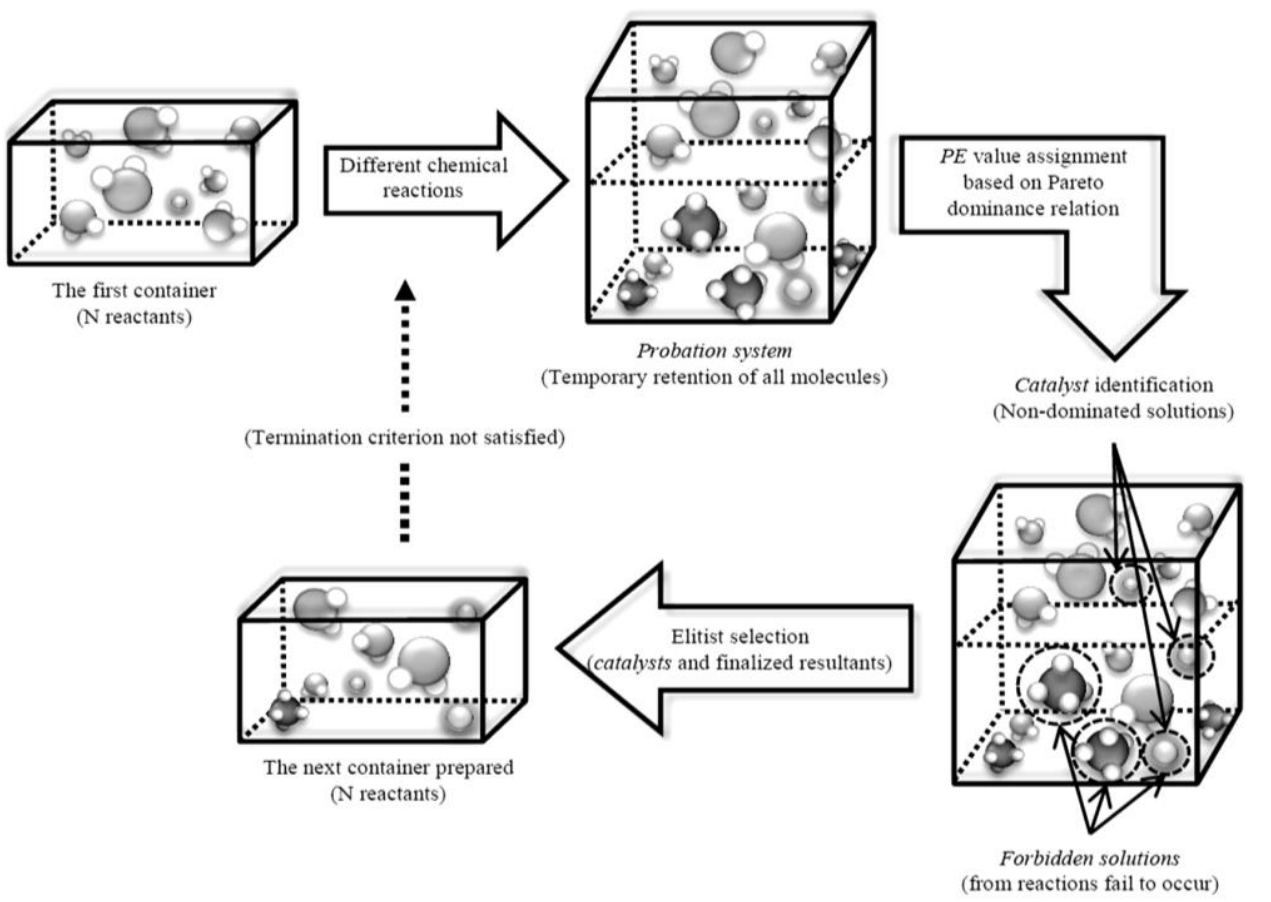

Fig. 2. Offspring generation in NSCRO

Table 4

Comparison between NSCRO and NSGA-II

\begin{tabular}{|c|c|c|c|}
\hline & NSGA-II & NSCRO \\
\hline \multicolumn{2}{|l|}{ Inspiration } & Natural evolutionary process & $\begin{array}{l}\text { Chemical reactions proceed in the direction of } \\
\text { stability (towards lower energy) through } \\
\text { molecular collisions }\end{array}$ \\
\hline \multicolumn{2}{|l|}{ Principle } & $\begin{array}{llll}\begin{array}{l}\text { Natural } \\
\text { genetic }\end{array} & \text { selection and natural } \\
\end{array}$ & Energy conservation and energy redistribution \\
\hline \multicolumn{2}{|l|}{ Basic unit } & Chromosome & Molecule \\
\hline \multicolumn{2}{|c|}{ Solution search } & Population-based & Population-based \\
\hline \multicolumn{2}{|c|}{ Size of population } & $\begin{array}{l}\text { The number of solutions in the } \\
\text { parent population is more than one } \\
\text { and fixed, the size of offspring } \\
\text { population is identical to the size of } \\
\text { the parent population. }\end{array}$ & $\begin{array}{l}\text { The number of solutions in the parent } \\
\text { population (i.e., reactant population) is more } \\
\text { than one and fixed, the size of offspring } \\
\text { population (i.e., resultant population) can be } \\
\text { different from that of the parent population. }\end{array}$ \\
\hline \multirow{2}{*}{$\begin{array}{l}\text { Solution } \\
\text { quality } \\
\text { evaluation }\end{array}$} & Criterion & \multicolumn{2}{|c|}{$\begin{array}{l}\text { Both quality and diversity are considered, wherein the solution with the lower (better) } \\
\text { Pareto rank is preferred, or the solution located in a lesser crowded region within the same } \\
\text { Pareto front is preferred }\end{array}$} \\
\hline & Approach & $\begin{array}{l}\text { Fitness function not available, } \\
\text { based on non-dominance and } \\
\text { crowding distance comparisons }\end{array}$ & $\begin{array}{l}\text { Fitness value is required for implementation of } \\
\text { operators }\end{array}$ \\
\hline \multicolumn{2}{|c|}{ Solution selection } & $\begin{array}{l}\text { Extrinsic fitness-based process } \\
\text { (e.g., tournament selection) }\end{array}$ & $\begin{array}{l}\text { Intrinsically controlled by energy management } \\
\text { laws, performed within the operators }\end{array}$ \\
\hline \multirow[b]{3}{*}{ Operators } & Diversification & Crossover & Decomposition, Synthesis \\
\hline & Intensification & Mutation & $\begin{array}{l}\text { On-wall ineffective collision, Intermolecular } \\
\text { ineffective collision }\end{array}$ \\
\hline & Process design & $\begin{array}{l}\text { Applied in sequence within an } \\
\text { iteration }\end{array}$ & $\begin{array}{l}\text { Hyper-heuristic (Bargaoui et al., 2016), the } \\
\text { algorithm itself controls the operations to be } \\
\text { realized according to a given situation, only } \\
\text { one operator applied within an iteration }\end{array}$ \\
\hline
\end{tabular}


To visualize the whole process of offspring generation, an illustration is presented in Figure 2. Within each chemical processing iteration, a predefined number of molecules are put into a container to undergo a series of chemical reactions. Hypothetically, reactants and temporary resultants are allowed to coexist in a probation system.

After each molecule is assigned a $P E$ value, the realization of chemical reactions continues to complete by producing finalized resultants and removing forbidden solutions. After each molecule is assigned a $P E$ value, the realization of chemical reactions continues to complete by producing finalized resultants and removing forbidden solutions. Meanwhile, a certain number of catalysts are identified and assumed to be produced additionally. To prepare for future reactions, both the catalysts and sequentially selected finalized resultants are extracted from the current iteration. Before ending this section, we compare the characteristic differences between two metaheuristics in Table 4.

\section{NUMERICAL RESULTS}

\subsection{Performance measure}

The performance of multi-objective metaheuristics is not as straightforward to evaluate as mono-objective methods. The comparison between different sets of Pareto solutions requires comprehensive assessments of solution quality through various quantitative measures. The definitions of dominance relations are well-known and need not be repeated here. Readers are welcome to consult Zitzler et al. (2003).

In this study, the hypervolume indicator (HV) (Zitzler and Thiele, 1998) is adopted to evaluate the quality of Pareto front approximations produced by NSGA-II and NSCRO. This measure considers the proportion of the objective space dominated by the obtained Pareto front approximation. This metric provides a qualitative measure of convergence as well as diversity in a combined sense (Talbi, 2009). The HV metric is selected because it is the only unary indicator that possesses Pareto compliance (Riquelme et al., 2015), and it is deemed as one of the existing indicators that at best allows inferring that an approximation set is not worse than another (Zitzler et al., 2003). Since this metric is free from the arbitrary scaling of objective values, normalized objective function values are used to eliminate such difficulty. The same reference point is selected as the nadir point of the Pareto front approximations obtained from all runs by both algorithms (Auger et al., 2009). Hence, a larger value of HV means better performance.

\subsection{Parameter settings}

The parameter settings of a metaheuristic can have a profound impact on its performance and tuning parameters can be a difficult task because the optimal parameter setting is case dependent. In the single objective CRO, energy values relate closely to objective function values, which vary significantly between problems and hence fixed parameter values may not exert their best effects on optimization problem. In NSCRO, the proposed $P E$ assignment is based on Pareto dominance relation, which is scale-invariant and helps ease this situation. One test scenario (i.e., the tri-objective under low demand with a budget equal to 40,000) is selected for the parameter tuning for both NSCRO and NSGA-II. 20 random seed lists are used for testing. To evaluate the general performance of the metaheuristics, the average value of the HVs obtained is compared for determining the best combination of parameter values.

In NSCRO, the specific parameters include six parameters [ $N$, initial KE, MoleColl, KELossRate, $\alpha, \beta]$, and the initial combination of their values was set to [200, 1.0, $0.5,0.6,20,0.5]$. During the tuning, with the values of other parameters fixed, the population size $N$ was first varied and the best value found was 100. Then, the parameter combination was changed to $[100,1.0,0.5,0.6$, $20,0.5]$. Then, the value of the second parameter, initial $K E$, in this new combination was tuned while other values are fixed. This process repeated until all the parameter values were determined. The parameter settings are tuned as [100, $4,0.6,0.7,10,3.5]$, which will be used in all the test scenarios presented later. For the parameters in NSGA-II, the same tuning method was adopted and the values for population size, crossover probability, and mutation probability was found to be $100,0.8$, and 0.01 , respectively.

In order to provide a fair comparison, the same frequency of solving the lower level problem (25,000 times) was used as a termination criterion.

\subsection{Trade-offs among three objectives}

In this study, the Pareto front approximations of the formulated tri-objective BTPE are obtained for three test scenarios each, namely the low demand condition, the average demand condition, and the high demand condition. Their demand matrices are obtained by multiplying the original hourly O-D flow matrix by $0.5,1.0$, and 2.0, respectively. We presume that such variations respectively represent the idle, average, and rush hours to some extent, due to the lack of more detailed information of commuting O-D pairs. Each of the Pareto front approximations is depicted in a three-dimensional scatter plot with a narrow surface and three two-dimensional plots. The results are illustrated in Figure 3, Figure 4, and Figure 5, respectively. 

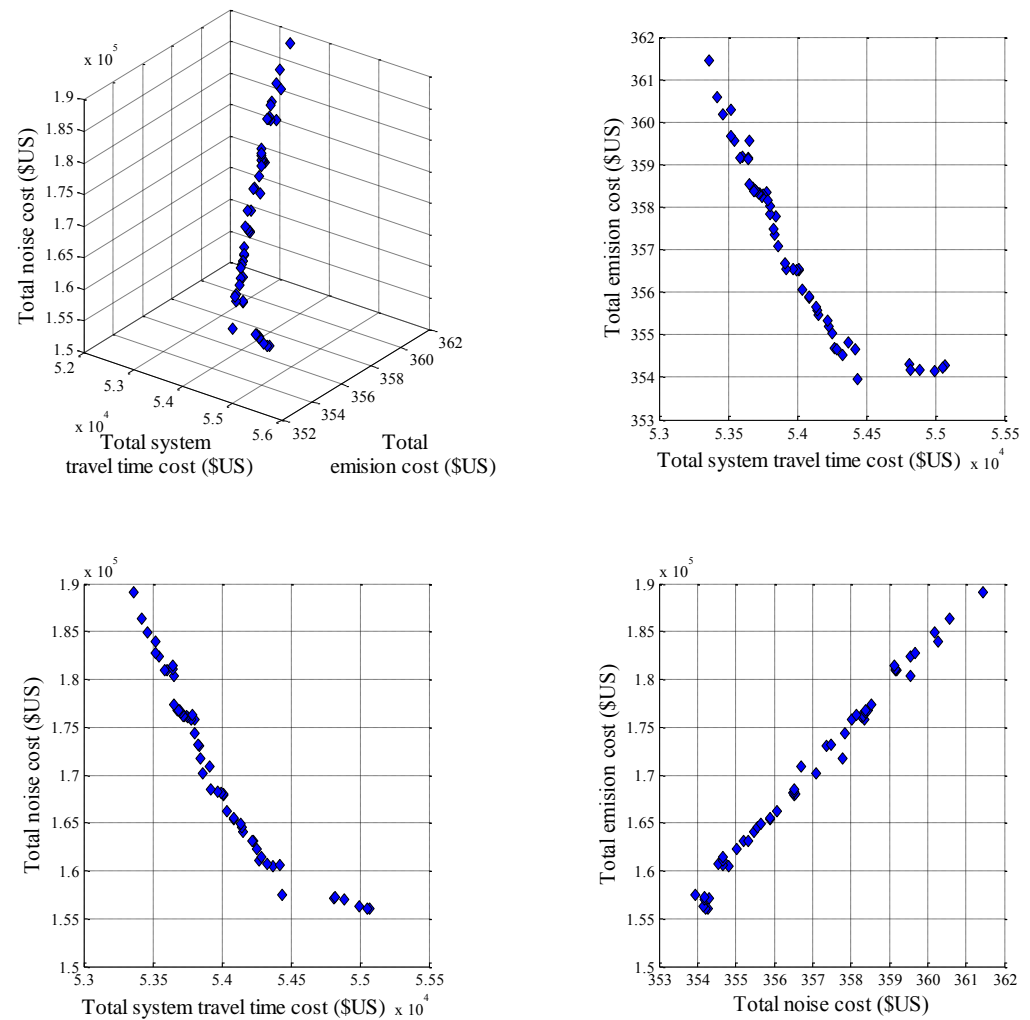

Fig. 3. Pareto non-dominated front for the low demand scenario
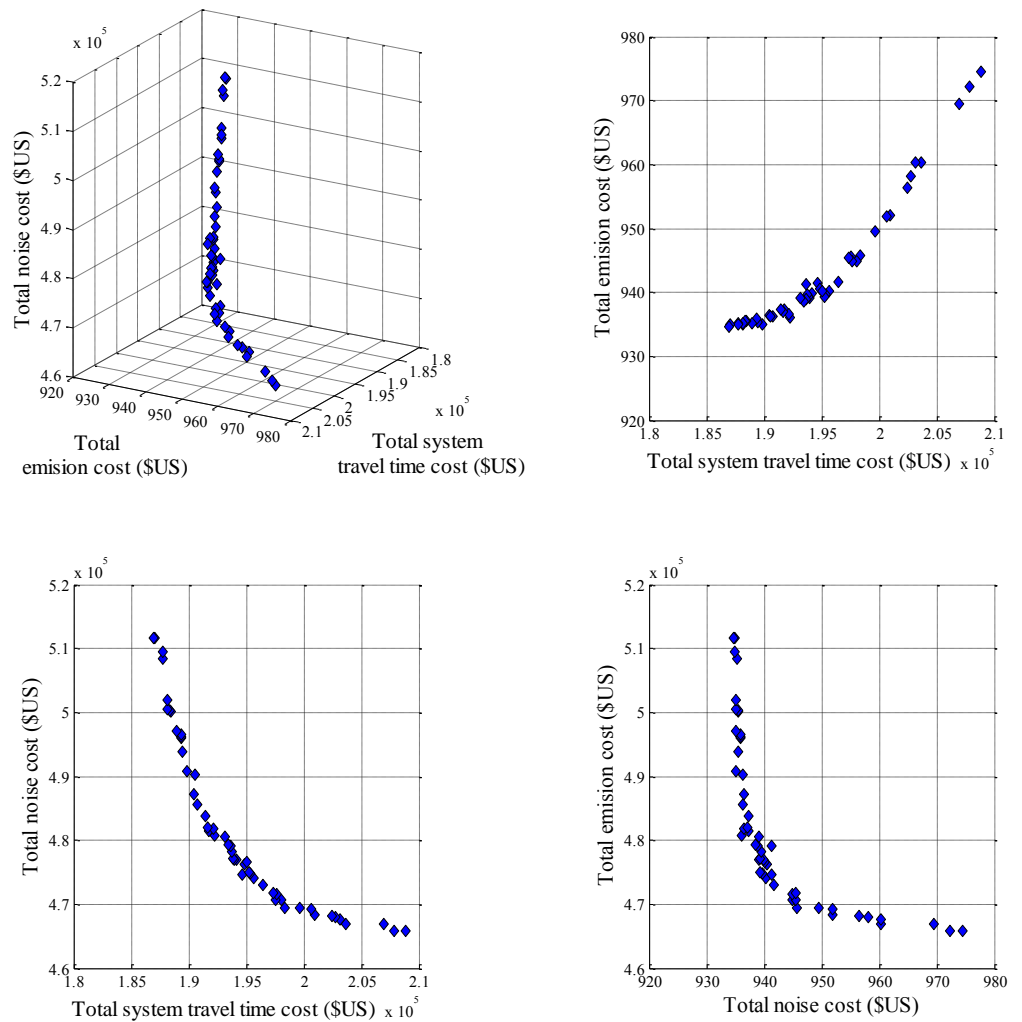

Fig. 4. Pareto non-dominated front for the average demand scenario 

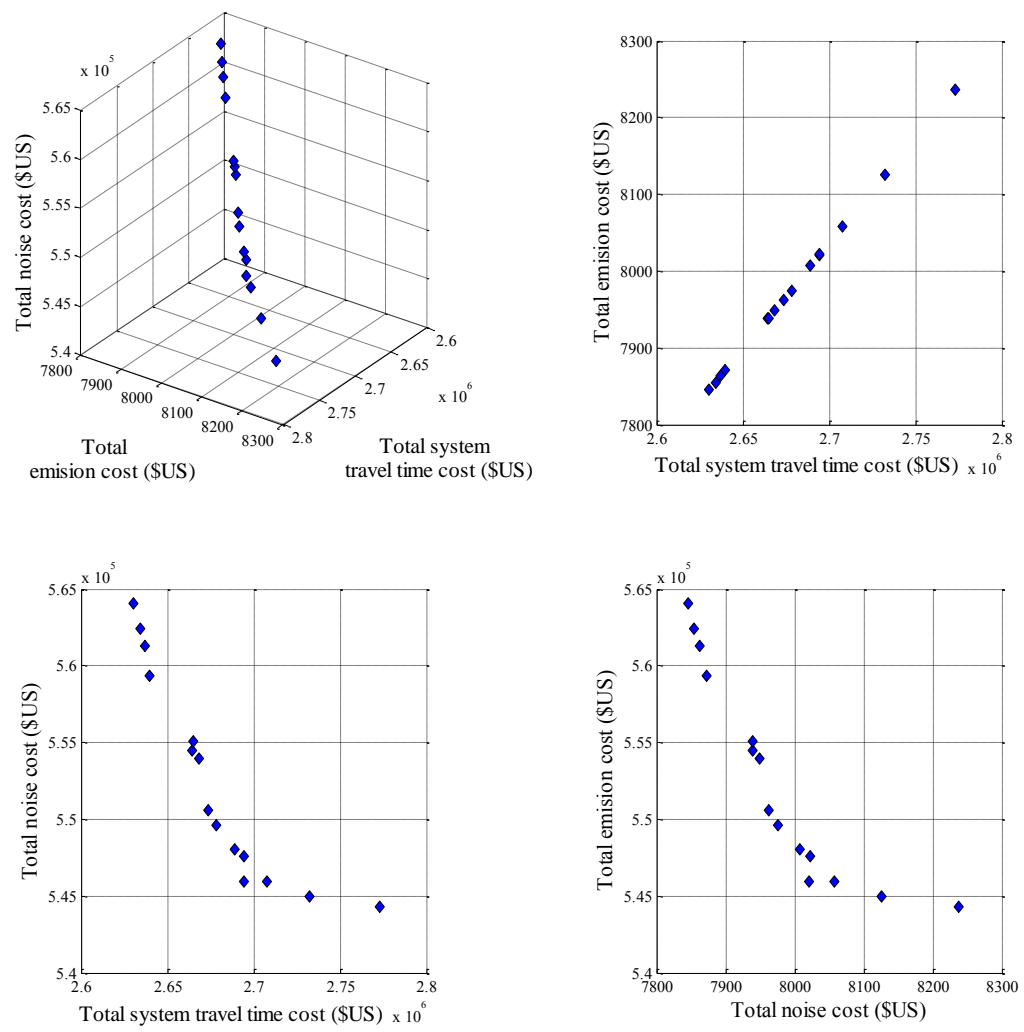

Fig. 5. Pareto non-dominated front for the high demand scenario

In the low demand test scenario, there are evident tradeoffs between minimizing vehicular emissions and congestion, as well as minimizations of excessive noise and congestion. In contrast, the relation between vehicular emissions and excessive noise possesses a trend of being aligned to some extent. When demand is low, speed is generally high. Hence, the improvement schemes that lower the travel speed show an improvement of both emissions and noise. Nevertheless, more than one optimal solution can be observed from the two-dimensional plot of total emission cost and total noise cost, which implies that these objectives cannot be simultaneously minimized by a single solution and trade-off exists.

When the travel demand reaches the average level, some different observations on conflicting relations are obtained. The result above shows that total emission cost starts to change in the same direction with total system travel time cost while minimizing total noise cost continues to conflict with minimizing total system travel time cost. To mitigate congestion or lower total system travel time cost, the traffic flow from congested routes is redirected onto the other routes with available capacity after the network is improved, resulting in higher travel speeds associated with relatively fewer vehicular emissions produced. However, the total amount of excessive traffic noise possesses different extremum with regard to travel speed, resulting in different changing trends. Hence, with travel demand increasing, the congestion on roads tends to be more severe, which results in lower average travel speed. According to the calculation formulae of $L_{e q}$, the traffic sound pressure level reaches the lowest value with the relatively low value of average speed (e.g., 20ft/s), while total emissions and total travel time cost do not.

With further increase of travel demand (the high demand scenario in Figure 5), objectives of minimizing congestion and emissions become strongly aligned and both are opposite to the noise objective, because in the high demand situation, speeds are already low on most links, which means reducing congestion now can help speed rise again. Congestion alleviation in such already congested networks is to a certain extent beneficial for emissions, but at a certain point emissions increase again. Moreover, the approximated Pareto optimal hypersurface has several concavities, indicated by the $2 \mathrm{D}$ view plot on the lower-left corner of Figure 5. Thus, it is not possible for the weighted sum method to yield all of the Pareto optimal points in such circumstances. This demonstrates that Pareto optimization is necessary for solving the proposed multi-objective BTPE to depict the Pareto optimal set comprehensively.

By comparing Figure 3, Figure 4, and Figure 5, it is observed that the number of optimal solutions obtained decreases as demand increases. This is attributable to the fact that conflicting relations between objectives have been weakened with the increase of traffic flow, which is a 
prominent component in the estimations of all objectives. These phenomena emphasize again the significance of carrying out a comprehensive multi-objective network design, especially in conjunction with different demand situations. In general, for each scenario, a considerable number of Pareto non-dominated solutions are acquired, which enables the planners to have various choices in the decision-making process.

\subsection{Solution performance comparison}

This section presents the computational experiments performed to assess the performance of the newly proposed NSCRO, as well as to compare it with the famous NSGA-II and the brute force method. All algorithms were coded by Intel(R) Visual Fortran within Microsoft Visual Studio 2010 framework. Tests were performed on a computer with an Intel(R) Core(TM)2 Quad CPU Q9650 @3.00GHz and 4.00 GB RAM.

\subsubsection{Benchmark scenarios for the comparison between the brute force method, NSCRO, and NSGA-II}

Whether or not the solution algorithm can produce the true Pareto optimal set remains a question unless all the possible solutions are examined. To address this question, different benchmark scenarios that at most three links can be chosen to be improved under the low demand situation were constructed. A brute force method was applied to enumerate all the possible solutions $(73,266)$ in each scenario in order to obtain the true Pareto fronts. To compare the performance of the two metaheuristics with the exact method, 20 runs were performed for both NSCRO and NSGA-II using the same set of random seeds. The maximum number of Pareto optimal solutions obtained out of 20 runs by each algorithm is provided in Table 5 .

Table 5

Results of the benchmark scenarios

\begin{tabular}{|c|c|c|c|}
\hline \multirow{2}{*}{ Scenarios } & \multicolumn{3}{|c|}{$\begin{array}{c}\text { Number of Pareto optimal solutions } \\
\text { obtained }\end{array}$} \\
\hline & $\begin{array}{l}\text { Brute force method } \\
\text { (true Pareto front) }\end{array}$ & NSCRO & NSGA-II \\
\hline$T S T T$ vs. TEC & 12 & 9 & 2 \\
\hline$T S T T$ vs. $T N C$ & 19 & 15 & 2 \\
\hline$T N C$ vs. $T E C$ & 2 & 2 & 0 \\
\hline $\begin{array}{l}\text { TSTC, TEC, } \\
\text { and } T N C\end{array}$ & 19 & 9 & 0 \\
\hline
\end{tabular}

Although neither algorithm succeeds in obtaining the whole set of Pareto optimal solutions in each benchmark scenario, NSCRO converges significantly better than NSGA-II towards the Pareto optimal front. It is well known that exact methods guarantee the global optimality of the provided solution. However, when the problems are with large dimensions, wherein assessing all solutions would take years, metaheuristics are better choices for practical purposes.

4.4.2 Test scenarios for the comparison between NSCRO and NSGA-II

To adequately assess and compare the performance of NSCRO and NSGA-II in terms of the diversity and convergence of solving different multi-objective BTPEs, both bi-objective and tri-objective scenarios are tested.

According to the results of tri-objective optimization in Section 4.3, objectives are not always conflicting with each other in each scenario. The strong aligned relation between objectives (e.g., minimize TNC and TEC under low demand) leads to very limited Pareto non-dominated solutions. Due to the inadequacy for testing multi-objective algorithms of such scenarios, only the bi-objective instances with opposed objectives are tested for performance comparison. The bi-objective test scenarios are selected and numbered as follows: 1) TSTC vs. TEC under low demand, 2) TSTC vs. TNC under low demand, 3) TSTC vs. TNC under average demand, 4) TEC vs. TNC under average demand, 5) $T S T C$ vs. TNC under high demand, and 6) TEC vs. TNC under high demand. Two sets of budgets $(40,000$ and $70,000)$ are used for constructing different sizes of solution space.

In addition to the bi-objective instances, six tri-objective test scenarios are constructed: simultaneous minimization of three objectives (i.e., TSTC, TEC, and TNC) under low, average, and high demand situations, constrained by two different budgets.

For each algorithm and problem, 20 runs using the same set of random seeds were performed. For each run, the HV value was calculated. The numerical results with respect to HV values and CPU times of NSCRO and NSGA-II are summarized in

Table 6 and Table 7 for the bi-objective and tri-objective test scenarios, respectively. The better result with respect to each measure between two algorithms is emphasized in boldface.

Since the same frequency of solving the lower-level problem (25,000 times) was used for both algorithms as termination criteria, the CPU times consumed by both algorithms are almost the same. The computational time may not be adopted as a good performance metric, simply because it highly depends on how the codes are programmed (Lam and $\mathrm{Li}, 2010$ ). Nevertheless, NSGA-II consumes slightly fewer CPU times for solving every test scenario.

In addition to CPU times, it is observed that NSCRO obtains larger average HV values of the majority of test scenarios. The eight higher average values of HV obtained by NSCRO out of twelve bi-objective test scenarios imply that NSCRO allows a higher quality of Pareto front approximation to be achieved on average. Also, NSCRO possesses smaller standard deviations of $\mathrm{HV}$ values on 
solving most problems, which indicates that NSCRO can achieve more stable results than NSGA-II does. The maximum $\mathrm{HV}$ value corresponds to the best Pareto front approximation for each test scenario obtained by NSCRO or NSGA-II among their 20 runs and NSCRO performs slightly better. NSCRO continues presenting larger average
$\mathrm{HV}$ values and lower standard deviations on solving most tri-objective test scenarios. Moreover, based on the obtained maximum HV values for tri-objective problems, NSCRO is demonstrated to find better Pareto front approximations than NSGA-II out of 20 runs.

Table 6

Results and computation time of the bi-objective problems

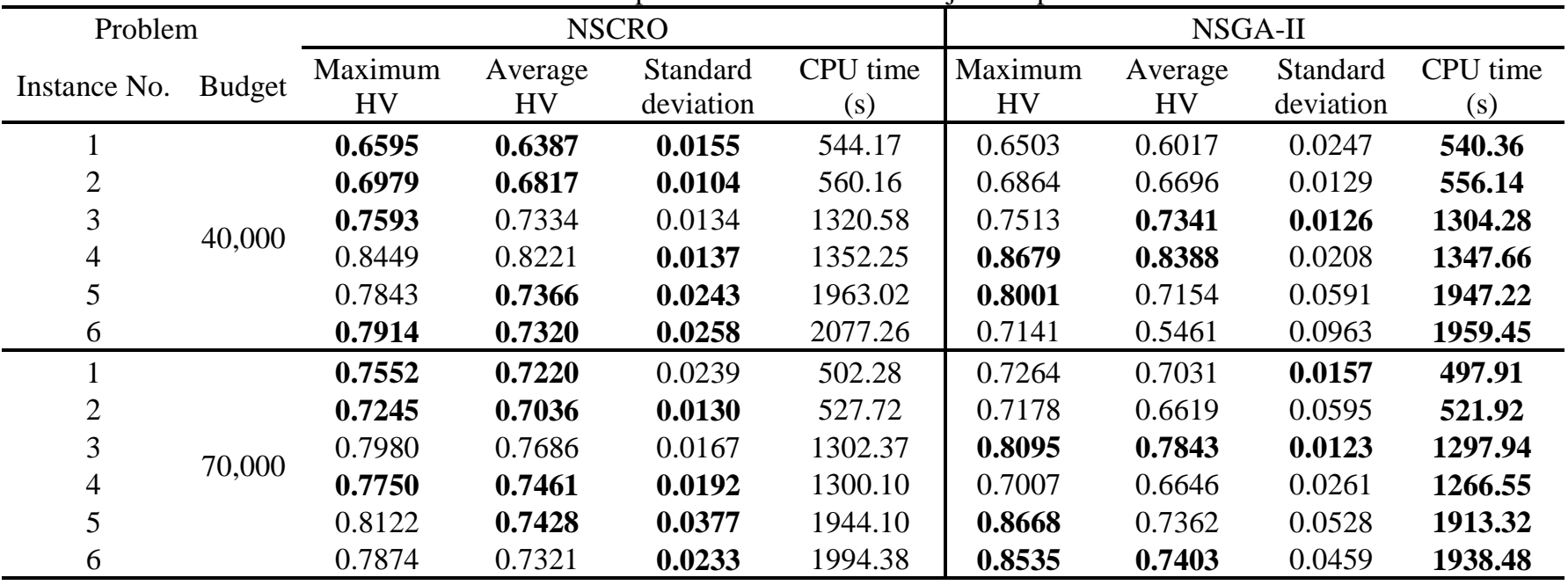

Table 7

Results and computation time of the tri-objective problems

\begin{tabular}{cccccc|cccc}
\hline \multirow{2}{*}{$\begin{array}{c}\text { Problem } \\
\text { Demand } \\
\text { conditions }\end{array}$} & Budget & $\begin{array}{c}\text { Maximum } \\
\text { HV }\end{array}$ & $\begin{array}{c}\text { Average } \\
\text { HV }\end{array}$ & $\begin{array}{c}\text { Standard } \\
\text { deviation }\end{array}$ & $\begin{array}{c}\text { CPU time } \\
(\mathrm{s})\end{array}$ & $\begin{array}{c}\text { Maximum } \\
\text { HV }\end{array}$ & $\begin{array}{c}\text { Average } \\
\text { HV }\end{array}$ & $\begin{array}{c}\text { Standard } \\
\text { deviation }\end{array}$ & $\begin{array}{c}\text { CPU } \\
\text { Time }(\mathrm{s})\end{array}$ \\
\hline 0.5D & & $\mathbf{0 . 6 0 6 6}$ & $\mathbf{0 . 5 9 3 6}$ & $\mathbf{0 . 0 0 9 5}$ & 606.19 & 0.5830 & 0.5427 & 0.0251 & $\mathbf{5 9 3 . 7 3}$ \\
1.0D & 40,000 & 0.7082 & 0.6925 & $\mathbf{0 . 0 1 0 6}$ & 1297.81 & $\mathbf{0 . 7 2 9 0}$ & $\mathbf{0 . 7 0 9 6}$ & 0.0173 & $\mathbf{1 2 7 1 . 7 1}$ \\
2.0D & & 0.6187 & $\mathbf{0 . 5 7 9 1}$ & $\mathbf{0 . 0 3 0 4}$ & 1987.89 & $\mathbf{0 . 7 1 1 8}$ & 0.5330 & 0.0906 & $\mathbf{1 8 8 5 . 2 2}$ \\
\hline 0.5D & & $\mathbf{0 . 6 3 9 1}$ & $\mathbf{0 . 5 9 3 4}$ & $\mathbf{0 . 0 2 8 6}$ & 568.47 & 0.6255 & 0.5744 & 0.0301 & $\mathbf{5 5 3 . 5 9}$ \\
1.0D & 70,000 & $\mathbf{0 . 7 4 5 9}$ & $\mathbf{0 . 7 2 6 4}$ & 0.0192 & 1302.55 & 0.7298 & 0.7126 & $\mathbf{0 . 0 1 2 8}$ & $\mathbf{1 2 8 1 . 7 9}$ \\
2.0D & & $\mathbf{0 . 7 5 8 7}$ & $\mathbf{0 . 6 8 4 1}$ & 0.0406 & 2014.72 & 0.6690 & 0.6288 & $\mathbf{0 . 0 2 5 0}$ & $\mathbf{1 9 7 2 . 0 4}$ \\
\hline
\end{tabular}

To provide a comprehensive evaluation of performance, the t-test was carried out on the difference between the average HV values obtained by NSCRO and NSGA-II for each test scenario. Based on HV values of 20 runs from both algorithms, the $p$-value of the following hypothesis was computed: $H_{0}$ : average $\mathrm{HV}$ value of algorithm NSCRO is equal to the average $\mathrm{HV}$ value of algorithm NSGA-II, and $H_{1}$ : average $\mathrm{HV}$ value of algorithm NSCRO is not equal to the average HV value of algorithm NSGA-II. With $95 \%$ confidence interval, if the $p$-value is found to be less than $0.05, H_{0}$ is rejected and $H_{1}$ is accepted. This allows us to verify that the average $\mathrm{HV}$ values obtained by NSCRO and NSGA-II are statistically different, and hence one outperforms the other. To present the results concisely, "s+" ("s-") is used to indicate that $H_{0}$ is rejected and that the algorithm performs significantly better (worse) than the other one, while " $\approx$ " means no conclusion on outperformance can be drawn. The summary of t-test results of the bi-objective and tri-objective problems are provided in Table 8 and Table 9, respectively.

According to the results in Table 8, NSCRO outperforms NSGA-II on solving six out of twelve bi-objective test scenarios, while NSGA-II outperforms NSCRO on solving one bi-objective test scenario only. It is worth noticing that for the test results of the four scenarios, wherein NSGA-II possess higher average $\mathrm{HV}$ values in Table 6 , we cannot reject most of the associated hypotheses (i.e., instances 3 and 4 with 40,000 budget and instance 6 with 70,000 budget). This indicates that just using the average value of the metric chosen is not adequate for evaluating the performance of metaheuristics due to the stochastic nature of the two algorithms. Compared with the bi-objective 
results, NSCRO performs significantly better than NSGA-II on obtaining better Pareto front approximations on a larger portion of tri-objective test scenarios.

Table 8

Results of t-test with $95 \%$ confidence interval on the difference in average $\mathrm{HV}$ values of bi-objective problems

\begin{tabular}{|c|c|c|c|}
\hline \multicolumn{2}{|c|}{ Problem } & \multirow[b]{2}{*}{ NSCRO } & \multirow[b]{2}{*}{ NSGA-II } \\
\hline $\begin{array}{l}\text { Instance } \\
\text { No. }\end{array}$ & Budget & & \\
\hline 1 & \multirow{6}{*}{40,000} & s+ & S- \\
\hline 2 & & s+ & S- \\
\hline 3 & & $\approx$ & $\approx$ \\
\hline 4 & & $\approx$ & $\approx$ \\
\hline 5 & & $\approx$ & $\approx$ \\
\hline 6 & & $\mathrm{~s}+$ & S- \\
\hline 1 & \multirow{6}{*}{70,000} & s+ & S- \\
\hline 2 & & s+ & S- \\
\hline 3 & & S- & S+ \\
\hline 4 & & $\mathrm{~s}+$ & S- \\
\hline 5 & & $\approx$ & $\approx$ \\
\hline 6 & & $\approx$ & $\approx$ \\
\hline
\end{tabular}

Table 9

Results of t-test with $95 \%$ confidence interval on the difference in average $\mathrm{HV}$ values of tri-objective problems

\begin{tabular}{ccccc}
\hline \multicolumn{2}{r}{ Problem } & & \multirow{2}{*}{ NSCRO } & \multirow{2}{*}{ NSGA-II } \\
\cline { 1 - 2 } Demand conditions & Budget & & $\mathrm{s}+$ & $\mathrm{s}-$ \\
$0.5 \mathrm{D}$ & & & $\mathrm{s}-$ & $\mathrm{s}+$ \\
$1.0 \mathrm{D}$ & \multirow{2}{*}{40,000} & & $\mathrm{~s}+$ & $\mathrm{s}-$ \\
\hline $0.0 \mathrm{D}$ & & & $\approx$ \\
$1.0 \mathrm{D}$ & \multirow{2}{*}{70,000} & $\mathrm{~s}+$ & $\mathrm{s}-$ \\
$2.0 \mathrm{D}$ & & $\mathrm{s}+$ & $\mathrm{s}-$ \\
\hline
\end{tabular}

To obtain more information on the level of convergence of both algorithms, an average convergence plot of the triobjective test scenario with low demand (budget: 40,000) over 20 runs is provided in Figure 6. For every certain number of lower level problem evaluations, the proportion of the objective space dominated by the Pareto front approximation (i.e., HV value) is determined by each algorithm. It is shown that NSGA-II converges relatively faster yet possibly to a local optimum, while NSCRO converges to a better Pareto front approximation within 30,000 lower level problem evaluations. It is indicated that NSCRO tends to perform better on global approximation and avoiding trapping into local optima. This analysis also demonstrates that 25,000 times of solving lower level problems used are appropriate as the stopping criteria for both algorithms.

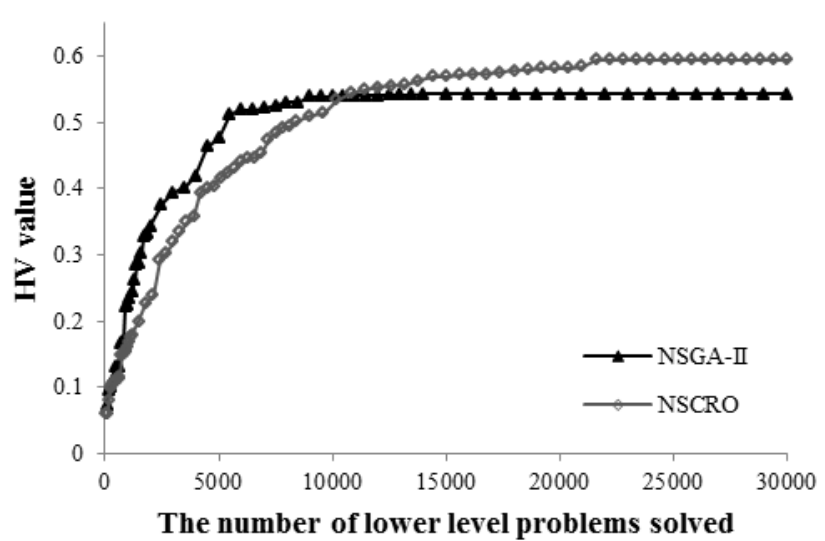

Fig. 6. Average convergence plots of NSCRO and NSGA-II

\subsubsection{Discussion}

In general, it can be deduced that NSCRO is able to obtain Pareto front approximations with higher quality in terms of diversity and convergence for the proposed BTPE. According to the No-Free-Lunch (NFL) theorem (Wolpert and Macready, 1997), NSCRO must perform comparably with other algorithms on average but can outperform other metaheuristics when matched to the right problem. Overall, NSCRO is demonstrated to be a more desirable solution algorithm for the proposed BTPE compared with NSGA-II.

As indicated by Xu et al. (2011), with appropriate control through parameter settings, CRO allows deployment of different operators to suit different stages of solution search, which enables it to enjoy the advantages of both GA and SA. Hence, it can be even seen as a hyper-heuristic since it controls the intensification and diversification adaptively (Bargaoui et al., 2016). Nevertheless, such merits highly depend on the parameter settings, and the number of parameters in NSCRO is relatively high among metaheuristics. It is believed that NSCRO requires much more efforts for the determination of parameter settings, which puts it at a disadvantage in the convenience for implementation. This may induce difficulty when it is applied for performing optimization on a large scale network, where a single time of solving the problem is computationally expensive. Moreover, the interrelationship between parameters is not clear, which may also influence the results of the tuning method chosen. Whether an adaptive or self-adaptive control of parameter settings can reduce the tuning efforts and further improve the performance is worth investigating.

\section{CONCLUSION}

This study contributes to the literature of BTPE study by proposing a tri-objective model using the Pareto optimization approach and implementing a multiobjective algorithm, named NSCRO to tackle the Pareto 
front approximating problem. Considering the macroscopic nature of network design problem, this study has made several assumptions and choices on the formulation of this multi-objective BTPE, such as single vehicle type (i.e., light duty vehicle), homogenous link performance function, and uniform housing density for the network. While we focus more on the trade-offs among objectives, the net effect of our simplifications and omissions is not obvious. Although some of the omissions (e.g., heavy duty vehicles) result in an underestimation of externalities, other assumptions (i.e., uniform housing density) might have the opposite effect. Nevertheless, there is no conceptual difficulty to generalize formulation by taking detailed heterogeneity into consideration for more accurate estimates, whenever detailed information (e.g., roads types, vehicle classes, freight demand, housing data, etc.) are available.

Numerical studies are provided to further investigate the interactions between objectives under different travel demand situations. The performance of newly proposed NSCRO is evaluated and compared with NSGA-II and the brute force method. The main findings and implications of this study are summarized:

- The distribution of the Pareto non-dominated solutions of this proposed tri-objective BTPE endorses the conclusion in Szeto et al. (2014) that external environmental costs caused by the traffic cannot always be minimized with the total system travel costs at the same time. Hence, it is important for transportation network planners to incorporate the environmental costs in the NDP and minimize them.

- The trade-offs between different objectives are explicitly illustrated. Furthermore, it is demonstrated that with low travel demand, total emission cost or total noise cost conflicts more with total system travel time cost, rather than with each other, respectively. In the average and high demand situations, total system travel time cost does not conflict with total emission cost while total noise cost conflicts with both total system travel time cost and total emission cost. The number of Pareto optimal solutions obtained abates with increasing travel demand. Transportation network planners need to weigh and consider the trade-offs more carefully when improving transportation networks with regard to different demand situations.

- A multi-objective meta-heuristic NSCRO is proposed and implemented for the optimal design of the bi-objective and tri-objective BTPE. The comparative results from the Sioux Falls network demonstrate that NSCRO outperforms NSGA-II on acquiring better Pareto front approximations of most test scenarios. Such optimistic results prove that there is no harm to implement NSCRO for solving bi-level multi-objective network design problems.
A few future research directions can be drawn from this study. The objectives in this BTPE account for the aggregate network travel time, emissions, and excessive noise, and this may lead to unbalanced travel time, emissions, or excessive noise levels throughout the network. Using equity measures in the objective function can ensure more equitable benefit to be introduced to various users in the network. Also, this study only considers the travel times, vehicular emissions, and excessive noise along street segments but ignores the delays occurred at intersections. Intersections not only induce delays but also may significantly contribute to the emissions and excessive noise because of excessive alteration of modal activities (e.g., starts, stops, acceleration, etc.) near intersections. Besides, the BPR function adopted suffers from its incompetence of travel time estimation in over-saturated traffic conditions. The inclusion of intersection and its influence on travel times and externalities as well as more realistic delay model can lead to more realistic estimates in a BTPE. Finally, the efficiency of the proposed algorithm can be further improved by parallel computing, based on the idea of multiple containers of Lam and $\mathrm{Li}$ (2010).

\section{ACKNOWLEDGMENTS}

The authors would like to thank the Editor and the seven anonymous reviewers for their constructive comments and valuable suggestions to improve the quality of the article. This research was supported by a grant from the National Natural Science Foundation of China (71271183).

\section{REFERENCES}

Adeli, H. \& Cheng, N.-T. (1993). Integrated genetic algorithm for optimization of space structures. Journal of Aerospace Engineering, 6(4), 315-328.

Adeli, H. \& Cheng, N.-T. (1994a). Augmented Lagrangian genetic algorithm for structural optimization. Journal of Aerospace Engineering, 7(1), 104-118.

Adeli, H. \& Cheng, N.-T. (1994b). Concurrent genetic algorithms for optimization of large structures. Journal of Aerospace Engineering, 7(3), 276-296.

Adeli, H., \& Hung, S. L. (1994). Machine learning: neural networks, genetic algorithms, and fuzzy systems. John Wiley \& Sons, Inc., New York.

Adeli, H., \& Kumar, S. (1995a). Distributed genetic algorithm for structural optimization. Journal of Aerospace Engineering, 8(3), 156-163.

Adeli, H., \& Kumar, S. (1995b). Concurrent structural optimization on massively parallel supercomputer. Journal of Structural Engineering, 121(11), 1588-1597.

Adeli, H. and Kumar, S. (1999). Distributed Computer-Aided Engineering for Analysis, Design, and Visualization, CRC Press, Boca Raton, Florida. 
Adeli, H., \& Sarma, K. C. (2006). Cost optimization of structures: fuzzy logic, genetic algorithms, and parallel computing. John Wiley \& Sons, West Sussex, United Kingdom.

Akinyemi, E. O., \& Zuidgeest, M. H. (2002). Managing transportation infrastructure for sustainable development. Computer-Aided Civil and Infrastructure Engineering, 17(3), 148-161.

Auger, A., Bader, J., Brockhoff, D., \& Zitzler, E. (2009, January). Theory of the hypervolume indicator: optimal $\mu$ distributions and the choice of the reference point. In Proceedings of the tenth ACM SIGEVO workshop on Foundations of genetic algorithms (pp. 87-102). ACM.

Bai, Q., Ahmed, A., Li, Z., \& Labi, S. (2015). A hybrid Pareto frontier generation method for trade-off analysis in transportation asset management. Computer-Aided Civil and Infrastructure Engineering, 30(3), 163-180.

Banister, D. (2007). Sustainable transport: challenges and opportunities. Transportmetrica, 3(2), 91-106.

Bargaoui, H., Driss, O. B., \& Ghédira, K. (2016, November). Minimizing makespan in multi-factory flow shop problem using a chemical reaction metaheuristic. In Evolutionary Computation (CEC), 2016 IEEE Congress on (pp. 29192926). IEEE.

Bar-Gera, H. (2016). Transportation networks, https://github.com/bstabler/TransportationNetworks

(Accessed on Jan 5, 2017).

Bechikh, S., Chaabani, A., \& Said, L. B. (2015). An efficient chemical reaction optimization algorithm for multiobjective optimization. IEEE Transactions on Cybernetics, 45(10), 2051-2064.

Benedek, C. M., \& Rilett, L. R. (1998). Equitable traffic assignment with environmental cost functions. Journal of Transportation Engineering, 124(1), 16-22.

Bouzoubia, S., Layeb, A., \& Chikhi, S. (2014). A multiobjective chemical reaction optimisation algorithm for multi-objective travelling salesman problem. International Journal of Innovative Computing and Applications, 6(2), 87-101.

Brands, T., \& Berkum, E. C. van (2014). Performance of a genetic algorithm for solving the multi-objective, multimodel transportation network design problem. International Journal of Transportation, 2(1), 1-20.

Cha, Y. J., \& Buyukozturk, O. (2015). Structural damage detection using modal strain energy and hybrid multiobjective optimization. Computer-Aided Civil and Infrastructure Engineering, 30(5), 347-358.

Chaabani, A., Bechikh, S., \& Ben Said, L. (2014, July). An indicator-based chemical reaction optimization algorithm for multi-objective search. In Proceedings of the Companion Publication of the 2014 Annual Conference on Genetic and Evolutionary Computation (pp. 85-86). ACM.

Chen, A., \& Xu, X. (2012). Goal programming approach to solving network design problem with multiple objectives and demand uncertainty. Expert Systems with Applications, 39(4), 4160-4170.

Dantzig, G. B., Harvey, R. P., Lansdowne, Z. F., Robinson, D. W., \& Maier, S. F. (1979). Formulating and solving the network design problem by decomposition. Transportation Research Part B: Methodological, 13(1), 5-17.

Deb, K., Pratap, A., Agarwal, S., \& Meyarivan, T. A. M. T. (2002). A fast and elitist multiobjective genetic algorithm: NSGA-II. IEEE Transactions on Evolutionary Computation, 6(2), 182-197.

Delucchi, M. A., \& Hsu, S. L. (1996). The External Damage Cost of Direct Noise from Motor Vehicles (Report No. UCD-ITS-RR-96-3 (4)). Davis, CA, USA: Institution of Transportation Studies, University of California, Davis.

Duan, H., \& Gan, L. (2015). Orthogonal multiobjective chemical reaction optimization approach for the brushless DC motor design. IEEE Transactions on Magnetics, 51(1), 1-7.

Farahani, R. Z., Miandoabchi, E., Szeto, W. Y., \& Rashidi, H. (2013). A review of urban transportation network design problems. European Journal of Operational Research, 229(2), 281-302.

Ferguson, E. M., Duthie, J., \& Travis Waller, S. (2012). Comparing delay minimization and emissions minimization in the network design problem. Computer-Aided Civil and Infrastructure Engineering, 27(4), 288-302.

Goldberg, D. E. (1989). Genetic algorithms in search, optimization and machine learning. Reading: AddisonWesley.

Huang, B., Pan, Z., \& Wang, G. (2015). A methodology to control urban traffic noise under the constraint of environmental capacity: A case study of a double-decision optimization model. Transportation Research Part D: Transport and Environment, 41, 257-270.

Huang, K., Zhang, J., He, M., \& Zhu, J. (2009). Bi-level programming model of urban traffic network considering noise pollution control. In Proceedings of the 2nd International Conference on Transportation Engineering, Chengdu, China, 25-27, July (pp. 3399-3404).

Hung S.L. \& Adeli, H. (1994). A parallel genetic/neural network learning algorithm for MIMD shared memory machines. IEEE Transactions on Neural Networks, 5(6), 900-909.

Jia, P., Kato, H., \& Hayashi, Y. (2009). Road network optimization model with consideration of dynamic changes in long term evaluation for developing cities. In Proceedings of the 88th Transportation Research Board Annual Meeting (pp. 11-15).

Jiang, X. \& Adeli, H. (2008). Neuro-genetic algorithm for nonlinear active control of highrise buildings. International Journal for Numerical Methods in Engineering, 75(8), 770-786. 
Jiang, Y., \& Szeto, W. Y. (2015). Time-dependent transportation network design that considers health cost. Transportmetrica A: Transport Science, 11(1), 74-101.

Kim, B. J., \& Kim, W. (2006). An equilibrium network design model with a social cost function for multimodal networks. The Annals of Regional Science, 40(3), 473-491.

Kim, H., \& Adeli, H. (2001). Discrete cost optimization of composite floors using a floating-point genetic algorithm. Engineering Optimization, 33(4), 485-501.

Kociecki, M., \& Adeli, H. (2013). Two-phase genetic algorithm for size optimization of free-form steel spaceframe roof structures. Journal of Constructional Steel Research, 90, 283-296.

Kociecki, M., \& Adeli, H. (2014). Two-phase genetic algorithm for topology optimization of free-form steel space-frame roof structures with complex curvatures. Engineering Applications of Artificial Intelligence, 32, 218-227.

Kociecki, M., \& Adeli, H. (2015). Shape optimization of free-form steel space-frame roof structures with complex geometries using evolutionary computing. Engineering Applications of Artificial Intelligence, 38, 168-182.

Lam, A. Y. S., \& Li, V. O. K. (2010). Chemical-reactioninspired metaheuristic for optimization. IEEE Transactions on Evolutionary Computation, 14(3), 381399.

Lam, A. Y. S., Li, V. O. K., \& Wei, Z. (2012a). Chemical reaction optimization for the fuzzy rule learning problem. In Proceedings of IEEE Congress on Evolutionary Computation, Brisbane, Australia.

Lam, A. Y. S., Li, V. O. K., \& Yu, J. Q. (2012b). Real-coded chemical reaction optimization. IEEE Transactions on Evolutionary Computation, 16(3), 339 - 353.

Levinson, D., Gillen, D., Kanafani, A., \& Mathieu, J. M. (1996). The Full Cost of Intercity Transportation-A Comparison of High Speed Rail, Air and Highway Transportation in California. (Report No. UCB-ITS-RR96-3). Berkeley, CA, USA: Institute of Transportation Studies, University of California.

Li, H., Wang, L., \& Hei, X. (2015). Decomposition-based chemical reaction optimization (CRO) and an extended CRO algorithms for multiobjective optimization. Journal of Computational Science, 17, 174-204.

Li, Z. C., Lam, W. H., Wong, S. C., \& Sumalee, A. (2012). Environmentally sustainable toll design for congested road networks with uncertain demand. International Journal of Sustainable Transportation, 6(3), 127-155.

Linster, M. (1990). Background facts and figures. In: Transport Policy and the Environment, European Conference of Ministers of Transport (ECMT). European Conference of Ministers of Transport - OECD, Paris.

Litman, T. (2003). Transportation Cost and Benefit Analysis: Techniques, Estimates and Implications. Victoria Transport Policy Institute, Victoria, Canada.
Long, J., Szeto, W. Y., \& Huang, H. J. (2014). A bi-objective turning restriction design problem in urban road networks. European Journal of Operational Research, 237(2), 426439.

López, E., \& Monzón, A. (2010). Integration of sustainability issues in strategic transportation planning: a multi-criteria model for the assessment of transport infrastructure plans. Computer-Aided Civil and Infrastructure Engineering, 25(6), 440-451.

Ma, X., Jin, J., \& Lei, W. (2014). Multi-criteria analysis of optimal signal plans using microscopic traffic models. Transportation Research Part D: Transport and Environment, 32, 1-14.

Marler, R. T., \& Arora, J. S. (2010). The weighted sum method for multi-objective optimization: new insights. Structural and Multidisciplinary Optimization, 41(6), 853862.

Mathew, T. V., \& Sharma, S. (2006). Continuous network design with emission pricing as a bi-level optimization problem. In Proceedings of the Ninth International Conference on Applications of Advanced Technology in Transportation (pp. 804-809).

Matthews, H. S. (1999). The External Costs of Air Pollution and the Environmental Impact of the Consumer in the U.S. Economy (PhD thesis). Pittsburg, USA: Carnegie Mellon University.

Meng, Q., \& Yang, H. (2002). Benefit distribution and equity in road network design. Transportation Research Part B: Methodological, 36(1), 19-35.

Miandoabchi, E., Daneshzand, F., Szeto, W. Y., \& Farahani, R. Z. (2013). Multi-objective discrete urban road network design. Computers \& Operations Research, 40(10), 24292449.

Miandoabchi, E., Farahani, R. Z., \& Szeto, W. Y. (2012). Biobjective bimodal urban road network design using hybrid metaheuristics. Central European Journal of Operations Research, 20(4), 583-621.

Nagurney, A. (2000a). Congested urban transportation networks and emission paradoxes. Transportation Research Part D: Transport and Environment, 5(2), 145151.

Nagurney, A. (2000b). Sustainable Transportation Networks. Cheltenham: Edward Elgar Publishers.

Nagurney, A., Qiang, Q., \& Nagurney, L. S. (2010). Environmental impact assessment of transportation networks with degradable links in an era of climate change. International Journal of Sustainable Transportation, 4(3), 154-171.

Ng, M., \& Waller, S. (2009). Reliable system-optimal network design: convex mean-variance model with implicit chance constraints. Transportation Research Record: Journal of the Transportation Research Board, 2090, 68-74.

OECD (Organization for Economic Cooperation and Development) (1986). Environmental Effects of 
Automotive Transport, The OECD Compass Project, Organization for Economic Cooperation and Development, Paris.

Oh, B. K., Kim, D., \& Park, H. S. (2017). Modal response-based visual system identification and model updating methods for building structures. Computer-Aided Civil and Infrastructure Engineering, 32(1), 34-56.

Park, K., Oh, B. K., Park, H. S., \& Choi, S. W. (2015). GA-based multi-objective optimization for retrofit design on a multi-core PC cluster. Computer-Aided Civil and Infrastructure Engineering, 30(12), 965-980.

Passchier-Vermeer, W., \& Passchier, W. F. (2000). Noise exposure and public health. Environmental Health Perspectives, 108, 123-131.

Penic, M. A., \& Upchurch, J. (1992). TRANSYT-7F: Enhancement for fuel consumption, pollution emissions, and user costs. Transportation Research Record: Journal of the Transportation Research Board, 1360, 104-111.

Possel, B., Wismans, L. J., Van Berkum, E. C., \& Bliemer, M. C. (2010). The multi-objective network design problem using minimizing externalities as objectives: comparison of a genetic algorithm and simulated annealing framework. Transportation, 37 (1), 1-28.

Qin, J., Ni, L. L., \& Shi, F. (2013). Mixed transportation network design under a sustainable development perspective. The Scientific World Journal, 2013.

Qiu, Y., \& Chen, S. (2007). Bi-level programming for continuous network design of comprehensive transportation system based on external optimization. In Proceedings of 2007 IEEE International Conference on Grey Systems and Intelligent Services, GSIS 2007, Nanjing, China, 18-20, November (pp. 1186-1190).

Rafiei, M. H., \& Adeli, H. (2016). Sustainability in highrise building design and construction. The Structural Design of Tall and Special Buildings, 25(13), 643-658.

Rilett, L. R., \& Benedek, C. M. (1994). Traffic assignment under environmental and equity objectives. Transportation Research Record, 1443, 92-99.

Riquelme, N., Von Lücken, C., \& Baran, B. (2015, October). Performance metrics in multi-objective optimization. In Computing Conference (CLEI), 2015 Latin American (pp. 1-11). IEEE.

Sarma, K. \& Adeli, H. (2000a). Fuzzy genetic algorithm for optimization of steel structures. Journal of Structural Engineering, 126(5), 596-604.

Sarma, K. \& Adeli, H. (2000b). Fuzzy discrete multicriteria cost optimization of steel structures. Journal of Structural Engineering, 126(11), 1339-1347.

Sarma, K. C., \& Adeli, H. (2003). Data parallel fuzzy genetic algorithm for cost optimization of large space steel structures. International Journal of Space Structures, 18(3), 195-205.

Sarma, K.C. \& Adeli, H. (2001). Bi-level parallel genetic algorithms for optimization of large steel structures.
Computer-Aided Civil and Infrastructure Engineering, 16(5), 295-304.

Sarma, K.C. \& Adeli, H. (2002). Life-cycle cost optimization of steel structures. International Journal for Numerical Methods in Engineering, 55(12), 1451-1462.

Sharma, S., \& Mathew, T. V. (2011). Multiobjective network design for emission and travel-time trade-off for a sustainable large urban transportation network. Environment and Planning B: Planning and Design, 38(3), 520-538.

Siddique, N., \& Adeli, H. (2013). Computational intelligence: synergies of fuzzy logic, neural networks and evolutionary computing. John Wiley \& Sons, West Sussex, United Kingdom.

Siddique, N., \& Adeli, H. (2017). Nature-inspired chemical reaction optimisation algorithms. Cognitive Computation, $1-12$.

Srinivas, N., \& Deb, K. (1994). Muiltiobjective optimization using nondominated sorting in genetic algorithms. Evolutionary computation, 2(3), 221-248.

Steele, C. (2001). A critical review of some traffic noise prediction models. Applied Acoustics, 62(3), 271-287.

Sun, Y., Lam, A., Li, V. O., Xu, J., \& Yu, J. J. (2012), Chemical reaction optimization for the optimal power flow problem. In Proceedings of 2012 IEEE Congress on Evolutionary Computation (CEC) (pp. 1-8).

Szeto, W. Y., \& Wu, Y. (2011). A simultaneous bus route design and frequency setting problem for Tin Shui Wai, Hong Kong. European Journal of Operational Research, 209(2), 141-155.

Szeto, W. Y., Jaber, X. Q., \& Wong, S. C. (2012). Road network equilibrium approaches to environmental sustainability. Transport Reviews: A Transnational Transdisciplinary Journal, 32(4), 491-518.

Szeto, W. Y., Jiang, Y., Wang, D. Z. W., \& Sumalee, A. (2015). A sustainable road network design problem with land use transportation interaction over time. Networks and Spatial Economics, 15(3), 791-822.

Szeto, W. Y., Li, X., \& O’Mahony, M. (2008). Simultaneous occurrence of Braess' and emission paradoxes. In Proceedings of the 6th International Conference on Traffic and Transportation Studies (pp. 625-634).

Szeto, W. Y., Wang, Y., \& Wong, S. C. (2014). The chemical reaction optimization approach to solving the environmentally sustainable network design problem. Computer-Aided Civil and Infrastructure Engineering, 29(2), 140-158.

Talbi, E. G. (2009). Metaheuristics: from design to implementation (Vol. 74) (pp. 375). John Wiley \& Sons.

Tzeng, G. H., \& Chen, C. H. (1993). Multiobjective decision making for traffic assignment. IEEE Transactions on Engineering Management, 40(2), 180-187.

U.S. Bureau of Public Roads (1964). Traffic Assignment Manual. US Department of Commerce, Washington, D. C., United States. 
Ukkusuri, S. V., Mathew, T. V., \& Waller, S. T. (2007). Robust transportation network design under demand uncertainty. Computer-Aided Civil and Infrastructure Engineering, 22(1), 6-18.

Unnikrishnan, A., \& Lin, D. Y. (2012). User equilibrium with recourse: continuous network design problem. Computer-Aided Civil and Infrastructure Engineering, 27(7), 512-524.

Unnikrishnan, A., Valsaraj, V., Damnjanovic, I., \& Waller, S. T. (2009). Design and management strategies for mixed public private transportation networks: a meta-heuristic approach. Computer-Aided Civil and Infrastructure Engineering, 24(4), 266-279.

Vitins, B. J. \& Axhausen, K. W. (2009). Optimization of large transport networks using the ant colony heuristic, Computer-Aided Civil and Infrastructure Engineering, 24(1), 1-14.

Wallace, C. E., Courage, K. G., Hadi, M. A., \& Gan, A. G. (1998). TRANSYT-7F User's Guide. University of Florida, Gainesville, FL, USA.

Wang, H., Lam, W. H., Zhang, X., \& Shao, H. (2015). Sustainable transportation network design with stochastic demands and chance constraints. International Journal of Sustainable Transportation, 9(2), 126-144.

Wang, N., \& Adeli, H. (2014). Sustainable building design. Journal of Civil Engineering and Management, 20(1), 110 .

Wang, Y. \& Szeto, W. Y. (2017). Excessive noise paradoxes in urban transportation networks. Transportmetrica A: Transport Science, 13(3), 195-221.

Wismans, L. J. J., Berkum, E. C. van, \& Bliemer, M. C. J. (2011a). Comparison of multiobjective evolutionary algorithms for optimization of externalities by using dynamic traffic management measures. Transportation Research Record: Journal of the Transportation Research Board, 2263, 163-173.

Wismans, L. J. J., Berkum, E. C. van, \& Bliemer, M. C. J. (2011b, April). Comparison of evolutionary multi objective algorithms for the dynamic network design problem. In Networking, Sensing and Control (ICNSC), 2011 IEEE International Conference on (pp. 275-280). IEEE.

Wismans, L. J. J., Berkum, E. C. van, \& Bliemer, M. C. J. (2014a). Acceleration of solving the dynamic multiobjective network design problem using response surface methods. Journal of Intelligent Transportation systems, 18(1), 17-29.

Wismans, L. J. J., Berkum, E.C. van \& Bliemer, M. C. J. (2012, Oct). Externalities as objectives for DTM solving a dynamic multi-objective network design problem. In: 40th European Transport Conference, ETC 2012, Glasgow, Scotland, U.K. (pp. 1 - 17).

Wismans, L. J. J., Brands, T., Berkum, E. C. van, \& Bliemer, M. C. J. (2014b). Pruning and ranking the Pareto optimal set, application for the dynamic multi-objective network design problem. Journal of Advanced Transportation, 48(6), 588-607.

Wolpert, D. H., \& Macready, W. G. (1997). No free lunch theorems for optimization. IEEE transactions on evolutionary computation, 1(1), 67-82.

Xu, J., Lam, A. Y., \& Li, V. O. (2011). Chemical reaction optimization for task scheduling in grid computing. IEEE Transactions on Parallel and Distributed Systems, 22(10), 1624-1631.

Yin, Y., \& Lawphongpanich, S. (2006). Internalizing emission externality on road networks. Transportation Research Part D: Transport and Environment, 11(4), 292301.

Yu, J. J. Q., Li, V. O. K., \& Lam, A. Y. S. (2012). Sensor deployment for air pollution monitoring using public transportation system. In Proceedings of IEEE Congress on Evolutionary Computation, Brisbane, Australia.

Zhao, T., \& Gao, Z. (2006). The combined problem of the comprehensive discrete network design under environment constraints and optimal signal controls. Tumu Gongcheng Xuebao (China Civil Engineering Journal), 39, 102-106. (in Chinese)

Zitzler, E., \& Thiele, L. (1998, September). Multiobjective optimization using evolutionary algorithms - a comparative case study. In International Conference on Parallel Problem Solving from Nature (pp. 292-301). Springer Berlin Heidelberg.

Zitzler, E., Thiele, L., Laumanns, M., Fonseca, C. M., \& Da Fonseca, V. G. (2003). Performance assessment of multiobjective optimizers: An analysis and review. IEEE Transactions on Evolutionary Computation, 7(2), 117-132.

\section{APPENDIX}

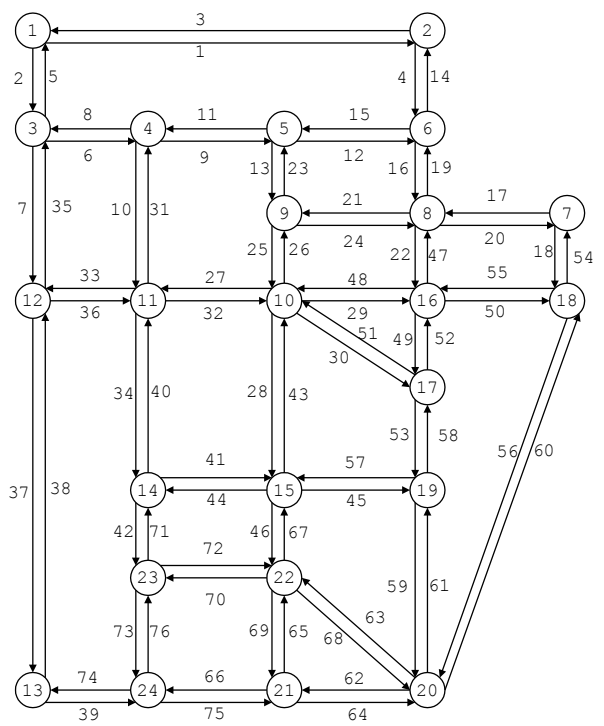

Network topology of Sioux Falls, South Dakota, United States 